CONFORMAL GEOMETRY AND DYNAMICS

An Electronic Journal of the American Mathematical Society

Volume 10, Pages 203-226 (September 21, 2006)

S 1088-4173(06)00119-6

\title{
FINITE SIMULTANEOUS BENDING
}

\author{
REZA CHAMANARA
}

\begin{abstract}
We construct a finite approximation to a Jordan curve with the given pair of bending measured laminations.
\end{abstract}

\section{INTRODUCTION}

Statement of the main result. This is the first in a series of papers about bending invariants of Jordan curves on the sphere. The content of this paper provides us with the basic approximation theorem to construct a Jordan curve on the sphere with the given pair of bending measured laminations on its pair of convex hull boundary components. The setting for the main theorem is as follows. Let $\mathbf{D}$ be the unit disk equipped with a complete metric of constant negative curvature -1 . For $\sigma= \pm, \mathcal{L}^{\sigma}$ is a finite lamination of $\mathbf{D}$, i.e., it is a finite collection of pairwise disjoint geodesics in $\mathbf{D}$. These geodesics are called the leaves of $\mathcal{L}^{\sigma}$. $E^{\sigma}$ denotes the set of the endpoints of the leaves of $\mathcal{L}^{\sigma}$ and $E$ denotes $E^{+} \cup E^{-} . \mathbf{D}^{*}$ denotes the complement of the closure of $\mathbf{D}$ in the sphere. For any finite lamination $\mathcal{L}$ on $\mathbf{D}, \mathcal{L}^{*}$ denotes the lamination on $\mathbf{D}^{*}$ whose leaves have the same endpoints as the leaves of $\mathcal{L}$. We assume that no two leaves share an endpoint. An external component of $\mathcal{L}^{\sigma}$ is a connected component of $\mathbf{D} \backslash \mathcal{L}^{\sigma}$ whose boundary contains only one leaf of $\mathcal{L}^{\sigma}$ and a leaf is called external if it is on the boundary of an external component. We assume that the pair $\left(\mathcal{L}^{+}, \mathcal{L}^{-}\right)$is fully binding, i.e. any external leaf intersects two external leaves of the other lamination (see Figure 4). Given a finite measured lamination $\mathcal{L}$ with the set $E$ of endpoints, any map $f: E \rightarrow \mathbf{S}^{1}$ that preserves the cyclic order of points, defines a new finite measured lamination denoted by $f(\mathcal{L})$. For any finite geodesic lamination with assignment of a weight $0<w(l)<\pi$ to each leaf $l, \mathfrak{G}_{\mathcal{L}}(\mathbf{D})$ is defined to be the Möbius structure on the disk obtained from the standard Möbius structure on $\mathbf{D}$ by cutting along all the leaves $l$ of $\mathcal{L}$ and gluing a lens of angle $w(l)$ to each of them. The natural extension of the developing map of this Möbius structure to $\overline{\mathbf{D}}$ defines a map $\mathcal{G}: \mathbf{S}^{1} \rightarrow \partial \mathcal{G}_{\mathcal{L}}(\mathbf{D})$. We assign the same weight $0<\vartheta<\pi$ to all leaves of the pair $\left(\mathcal{L}^{+}, \mathcal{L}^{-}\right)$. Then the finite simultaneous bending theorem states the following (see Figure 3i).

Theorem (Finite simultaneous bending). Given a pair $\left(\mathcal{L}^{+}, \mathcal{L}^{-}\right)$of finite laminations satisfying the conditions stated in the previous paragraph, there are

Received by the editors March 22, 2004 and, in revised form, April 3, 2006.

2000 Mathematics Subject Classification. Primary 51M15, 51B10; Secondary 51N25, 51M10, $30 \mathrm{~F} 40$.

Key words and phrases. Möbius structures, grafting, convex hulls, disk patterns.

This research was in part supported by a grant from IPM (No. 83510120).

(C)2006 American Mathematical Society Reverts to public domain 28 years from publication 
maps $f^{\sigma}: E \rightarrow \mathbf{S}^{1}$ preserving the cyclic order of points such that:

(1) The extensions of the developing maps of $\mathcal{G}_{f^{+}\left(\mathcal{L}^{+}\right)}(\mathbf{D})$ and $\mathcal{G}_{f^{-}\left(\mathcal{L}^{-}\right)^{*}}\left(\mathbf{D}^{*}\right)$ to $\overline{\mathbf{D}}$ and $\overline{\mathbf{D}}^{*}$ define homeomorphisms $\mathcal{G}^{+}: \mathbf{S}^{1} \rightarrow \partial \mathcal{G}_{f^{+}\left(\mathcal{L}^{+}\right)}(\mathbf{D})$ and $\mathcal{G}^{-}$: $\mathbf{S}^{1} \rightarrow \partial \mathcal{G}_{f^{-}\left(\mathcal{L}^{-}\right)^{*}}\left(\mathbf{D}^{*}\right)$.

(2) There is a Möbius map $F$ such that $\mathcal{G}_{f^{+}\left(\mathcal{L}^{+}\right)}\left(f^{+}(x)\right)=F\left(\mathcal{G}_{f^{-}\left(\mathcal{L}^{-}\right)^{*}}\left(f^{-}(x)\right)\right)$ for any $x \in E=E^{+} \cup E^{-}$.

Notice that the maps $\mathcal{G}_{f^{-}\left(\mathcal{L}^{-}\right)^{*}} \circ f^{-}$and $\mathcal{G}_{f^{+}\left(\mathcal{L}^{+}\right)} \circ f^{+}$are defined only up to post composition with a Möbius map and the theorem claims that restrictions of these two maps to $E$ are in the same Möbius equivalence class. Moreover the assumption on all weights being equal to the same number $\vartheta$ is necessary. We will also show that there is an interval $I$ and a family $\left(f_{\varrho}^{+}, f_{\varrho}^{-}\right), \varrho \in I$, of pairs of maps such that any pair $\left(f^{+}, f^{-}\right)$of maps that satisfies the first conclusion of the theorem must satisfy $f^{+}=M^{+} \circ f_{\varrho}^{+}$and $f^{-}=M^{-} \circ f_{\varrho}^{-}$, for some $\varrho \in I$ and Möbius self-maps $M^{+}$and $M^{-}$of $\mathbf{S}^{1}$ (see Remark 4.18).

Motivation. Now, we briefly describe how this theorem fits into the main goals of this series of papers. We regard the sphere $\mathbf{S}^{2}$ as the boundary of the 3-dimensional hyperbolic space $\mathbb{H}^{3}$. Given a parameterized Jordan curve $j: \mathbf{S}^{1} \rightarrow J \subset \mathbf{S}^{2}$, the convex hull $\mathcal{C}(J)$ of $J$ is defined to be the smallest convex subset of $\mathbb{H}^{3}$ that contains all the geodesics connecting pairs of points in $J$. The boundary of $\mathcal{C}(J)$ consists of two disks $\mathcal{D}^{+}$and $\mathcal{D}^{-}$. When equipped with the path metric inherited from $\mathbb{H}^{3}$, $\mathcal{D}^{+}$and $\mathcal{D}^{-}$are both isometric to the hyperbolic plane $\mathbb{H}^{2}$ (see [7]). Moreover, for $\sigma= \pm$, any isometry from $\mathcal{D}^{\sigma}$ onto $\mathbb{H}^{2}$, extends to $\overline{\mathcal{D}^{\sigma}}=\mathcal{D}^{\sigma} \cup J$ and defines a map $h^{\sigma}: J \rightarrow \mathbf{S}^{1}$. Different choices of isometries lead to post-composition of $h^{\sigma}$ with a Möbius self-map of $\mathbf{S}^{1}$. We call the class of the pair of maps $f^{\sigma}=h^{\sigma} \circ j: \mathbf{S}^{1} \rightarrow \mathbf{S}^{1}$, $\sigma= \pm$, up to post-composition of the two maps with (possibly different) Möbius self-maps of $\mathbf{S}^{1}$, the marking maps for $J$. For $\sigma= \pm, \mathcal{D}^{\sigma}$ is a pleated surface and its pleating locus defines a measured lamination $\mu^{\sigma}$ on the unit disk $\mathbf{D}$ regarded as a model of the hyperbolic plane.

Let $\mathcal{J}$ be the space of all parameterized Jordan curves on $\mathbf{S}^{2}$ modulo the action of $\operatorname{PSL}(2, \mathbb{C})$. We define $\Theta$ and $\Sigma$ to be the maps that send any $J \in \mathcal{J}$ respectively to the pairs $\left(\mu^{+}, \mu^{-}\right)$and $\left(f^{+}, f^{-}\right)$. The image of $\Sigma$ is defined up to the product action of Möbius self-maps of $\mathbf{S}^{1}$ on the two components. For a Möbius map $M$ and a Jordan curve $j: \mathbf{S}^{1} \rightarrow J, \mathcal{C}(M(J))=M(\mathcal{C}(J))$ and therefore $M \circ j$ and $j$ have the same $\Theta$ and $\Sigma$ images. The main goal of this series of papers is to show that $\Theta$ and $\Sigma$ define homeomorphic correspondences between certain subsets of $\mathcal{J}$ and specific subsets of their images.

One special case is when $J$ is the limit set of a quasi-Fuchsian group $\hat{\Gamma}$. In this case, one can fix a Fuchsian group $\Gamma_{0}$ and parametrization $j$ of the limit set $J$ can be chosen to satisfy $j^{-1} \circ \hat{\Gamma} \circ j=\Gamma_{0}$. Then, for $\sigma= \pm, \Gamma^{\sigma}=h^{\sigma} \circ \hat{\Gamma} \circ\left(h^{\sigma}\right)^{-1}$ defines a Fuchsian group and $\mathrm{e}^{ \pm}=\mathbb{H}^{2} / \Gamma^{\sigma}$ are the hyperbolic structures on the two convex core boundary components of the 3 -manifold $\mathbb{H}^{3} / \hat{\Gamma}$. The maps $f^{\sigma}$ can be regarded as the extensions to $\mathbf{S}^{1}$ of the lifts of the quasi-conformal marking maps from the base surface $\mathbb{H}^{2} / \Gamma_{0}$ to $\mathbb{H}^{2} / \Gamma^{\sigma}$. Moreover $\mu^{+}$and $\mu^{-}$are the lifts of the bending measured laminations of the two convex core boundary components of the 3-manifold $\mathbb{H}^{3} / \hat{\Gamma}$. Both maps $\Theta$ and $\Sigma$ have been studied extensively while many questions remain unanswered (see [2, 4], 6], 12, [13, 17, [18). One of the main goals in this series of papers is to answer some of these questions and 
also understand some of the known results in a different way which does not use the hyperbolic geometry of the 3-manifold and the corresponding quasi-Fuchsian group. This, in a way, is similar to the treatment of the earthquake theorem in [21] or the proof of the simultaneous uniformization in [1] that do not require the group invariance of the given circle homeomorphism or the two conformal structures.

In this paper we take the first step in understanding the image of the map $\Theta$. In other words, we are interested to know if for a given pair $\left(\mu^{+}, \mu^{-}\right)$of measured laminations, there is a Jordan curve that realizes this pair as its pair of bending laminations. Although we won't be able to answer this question in the stated setting, we will give an answer to a finite approximation to the problem.

A Jordan curve $J$, defines a pair of projective structures $\mathcal{P}^{+}$and $\mathcal{P}^{-}$on its two complementary components such that $\mathcal{G}_{f^{+}\left(\mu^{+}\right)}(\mathbf{D})=\mathcal{P}^{+}$and $\mathcal{G}_{-f^{-}\left(\mu^{-}\right)}(\mathbf{D})=$ $\mathcal{P}^{-}$, where $\mathcal{G}_{\mu}(\mathbf{D})$ denotes the grafting of $\mathbf{D}$ along $\mu$ and $\mathcal{G}_{-\mu}(\mathbf{D})$ is the projective structure on $\mathbf{D}$ defined by the complement of $\mathcal{G}_{\mu^{*}}\left(\mathbf{D}^{*}\right)$ in $\mathbf{S}^{2} . \mathcal{G}_{-\mu}(\mathbf{D})$ is defined only if the complement of $\mathcal{G}_{\mu^{*}}\left(\mathbf{D}^{*}\right)$ in $\mathbf{S}^{2}$ is homeomorphic to $\mathbf{D}$. $\Theta(J)=\left(\mu^{+}, \mu^{-}\right)$ only if there are homeomorphisms $g^{+}$and $g^{-}$of $\mathbf{S}^{1}$ such that:

- The developing maps $d_{g^{+}}: \mathbf{D} \rightarrow \mathcal{G}_{g^{+}\left(\mu^{+}\right)}(\mathbf{D})$ and $d_{g^{-}}: \mathbf{D} \rightarrow \mathcal{G}_{-g^{-}\left(\mu^{-}\right)}(\mathbf{D})$ are homeomorphisms and they extend to homeomorphisms $\bar{d}_{g^{+}}: \mathbf{D} \cup \mathbf{S}^{1} \rightarrow$ $\overline{\mathcal{G}_{g^{+}\left(\mu^{+}\right)}(\mathbf{D})}$ and $\bar{d}_{g^{-}}: \mathbf{D} \cup \mathbf{S}^{1} \rightarrow \overline{\mathcal{G}_{-g^{-}\left(\mu^{-}\right)}(\mathbf{D})}$ (both defined only up to post composition with a Möbius map).

- $\left(\bar{d}_{g^{-}} \circ g^{-}\right) \circ\left(\bar{d}_{g^{+}} \circ g^{+}\right)^{-1}$ is equal to restriction of a Möbius map.

The finite approximation theorem proven in this paper considers the case with $\mu^{+}$and $\mu^{-}$having only finitely many leaves and it also checks for $\bar{d}_{g^{-}} \circ \bar{d}_{g^{+}}^{-1}$ being equal to a Möbius map only on the finite set of the endpoints of the leaves of $\mu^{+}$and $\mu^{-}$. In this sense our approach is similar to the proof of the earthquake theorem given in [8].

Outline of the paper. In the first section we introduce the notation and terminology for finite measured laminations and weighted planar trees. In section 2 we introduce the definitions required to state the finite simultaneous bending theorem. In section 3 we introduce certain configurations of disks, called complexes of disks, which are parameterized by pairs of weighted planar trees. We will show that the finite simultaneous bending theorem is equivalent to an existence theorem for complexes of disks. In section 4 we show that the existence of complexes of disks is equivalent to the existence of certain maximal ideal disk patterns. In particular we show that the finite simultaneous bending theorem follows from a version of Andreev's theorem proven by Igor Rivin in [14, [15], and [16]. In this section we also give example 4.4 to show that the assumption on the equality of the weights is necessary.

\section{Finite measured laminations and Planar trees}

Finite measured laminations. Let $\mathbf{D}$ be the unit disk equipped with a complete hyperbolic metric of constant curvature -1 . A finite geodesic lamination $\mathcal{L}$ on $\mathbf{D}$ is a finite union of pairwise disjoint geodesics in $\mathbf{D}$ (see Figure 1, left). Each of these geodesics is called a leaf of $\mathcal{L}$. A finite measured lamination is a finite geodesic lamination together with an assignment of a positive weight $w(l)$ to each leaf $l$. Given a finite measured lamination $\mathcal{L}$ with the set $E$ of endpoints, and a map $f: E \rightarrow \mathbf{S}^{1}$ that preserves the cyclic order of points, $f(\mathcal{L})$ is the finite measured 
lamination whose leaves connect the images under $f$ of pairs of endpoints of leaves of $\mathcal{L}$. For any leaf $l$ of $\mathcal{L}$, the weight assigned to the leaf $f(l)$ of $f(\mathcal{L})$ is equal to the weight assigned to $l$.

Let $\mathbf{D}^{*}$ be the complement of $\mathbf{D}$ in $\mathbf{S}^{2}$ equipped with a complete hyperbolic metric of constant curvature -1 . Any geodesic $l$ in $\mathbf{D}$ defines a geodesic $l^{*}$ in $\mathbf{D}^{*}$ with the same endpoints on $\mathbf{S}^{1}$. Given a finite measure lamination $\mathcal{L}$ in $\mathbf{D}$ with weights $w_{i}$ on leaves $l_{i}, 1 \leq i \leq n, \mathcal{L}^{*}$ is the finite measured lamination of $\mathbf{D}^{*}$ which is formed of leaves $l_{i}^{*}$ with weights $w_{i}$.
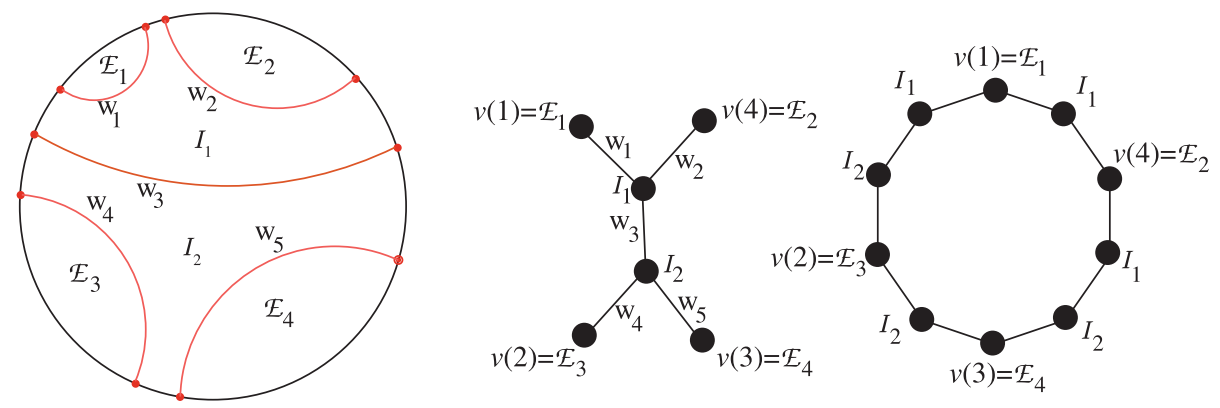

Figure 1. Left-hand side: A finite measured lamination. The red leaves are external and the only orange leaf is internal. Complementary components are labeled $I$ for internal and $E$ for external. Right-hand side: The corresponding weighted planar tree $\mathrm{T}(\mathcal{L})$ and the loop $\mathcal{C}(\mathrm{T}(\mathcal{L}))$ associated with it.

A connected component of $\mathbf{D} \backslash \mathcal{L}$ is called external if it contains exactly one leaf of $\mathcal{L}$ on its boundary; otherwise it is called internal. A leaf of $\mathcal{L}$ is called external if it is on the boundary of an external complementary component of $\mathcal{L}$; otherwise it is called internal (see Figure 1, left).

To any finite lamination $\mathcal{L}$ one can assign a weighted planar tree $\mathbb{T}(\mathcal{L})$ as follows (see Figure 1, middle). Any connected component of $\mathbf{D} \backslash \mathcal{L}$ is a vertex of $\mathbb{T}(\mathcal{L})$. A pair of vertices of $\mathbb{T}(\mathcal{L})$ are connected by an edge of weight $w$ if the corresponding connected components of $\mathbf{D} \backslash \mathcal{L}$ share a leaf $l$ of $\mathcal{L}$ on their boundaries and the weight on $l$ is $w$.

Planar trees. Let $\mathbb{T}$ be a planar tree. We define $\partial \mathbb{T}$ to be the set of all vertices of $\mathbb{T}$ with valence one. We denote the sub-tree generated by all the vertices of $\mathbb{T}$ which are not in $\partial \mathbb{T}$ by $\operatorname{int}(\mathbb{T})$. A rooted tree $(\mathbb{T}, v(1))$ is a tree with a choice of a vertex $v(1) \in \partial \mathbb{T}$. For a planar rooted tree $\mathbb{T}$, we define $\mathcal{C}(\mathbb{T})$ to be the loop defined as follows. We start at $v(1)$ and we travel on the tree according to the following rule: After arriving at any vertex $v$ through an edge $e$ we continue on the first edge connected to $v$ and to the right of $e$. If $v \in \partial \mathbb{T}$, there is only one edge $e$ connected to $v$, so we travel back along $e$. This way we have a loop $\mathcal{C}(\mathbb{T})$, where every vertex $v$ of $\mathbb{T}$ has appeared as many times as the valence of $v$ (see Figure 1, right). Suppose $N=\#(\partial \mathbb{T})$. Let $v(i), 1 \leq i \leq N$, be the vertices in $\partial \mathbb{T}$ labeled according to the order they appear in $\mathcal{C}(\mathbb{T})$. Denote the natural projection map from $\mathcal{C}(\mathbb{T})$ onto $\mathbb{T}$ by $\Pi$. For $i \in \mathbb{Z}_{N}$ and $v(i), v(i+1) \in \partial \mathbb{T}, \iota_{i}(\mathbb{T})$ denotes the image under $\Pi$ of all the vertices of $\mathcal{C}(\mathbb{T})$ that appear between $\Pi^{-1}(v(i))$ and $\Pi^{-1}(v(i+1))$. Every vertex $v$ in $\iota_{i}(\mathbb{T})$ has a unique pre-image $\Pi_{i}^{-1}(v)$ under $\Pi$ that lies between $v(i)$ and 
$v(i+1)$. For any $v \in \iota_{i}(\mathbb{T})$ that is not $\Pi(v(i+1))$ (respectively $\left.\Pi(v(i))\right)$, let $s_{i}(v)$ (respectively $p_{i}(v)$ ) be the image under $\Pi$ of the vertex in $\mathrm{C}(\mathbb{T})$ that appears right after (respectively right before) $\Pi_{i}^{-1}(v)$. For $v(i) \in \partial \mathbb{T}, p_{i-1}(v(i))=s_{i}(v(i))$ is the only vertex in $\operatorname{int}(\mathbb{T})$ which is connected to $v(i)$. For a vertex $v$, let $j(v)$ be the set of all the vertices connected to $v$.

Notice that for a finite measured lamination $\mathcal{L}$, the external complementary components of $\mathcal{L}$ correspond with the vertices in $\partial \mathbb{T}(\mathcal{L})$. By making a choice of an external complementary component of $\mathcal{L}, \mathbb{T}(\mathcal{L})$ turns into a rooted tree. Boundaries of the external complementary components of $\mathcal{L}$ intersect $\mathbf{S}^{1}$ in intervals that appear in the cyclic order compatible with the labeling of the vertices in $\partial \mathbb{T}(\mathcal{L})$ as described above. Let $N=\# \partial \mathbb{T}(\mathcal{L})>2$ and for $i \in \mathbb{Z}_{N}$ suppose $\mathcal{A}(i)$ and $\mathcal{A}(i+1)$ are two adjacent external complementary components of $\mathcal{L}$ which correspond with the vertices $v(i)$ and $v(i+1)$ of $\partial \mathbb{T}(\mathcal{L})$. $\mathbf{S}^{1} \backslash(\partial \mathcal{A}(i) \cup \partial \mathcal{A}(i+1))$ consists of two intervals, one of which does not intersect the boundary of any external complementary component of $\mathcal{L}$. Then the complementary components of $\mathcal{L}$ whose boundaries intersect this interval, together with $\mathcal{A}(i)$ and $\mathcal{A}(i+1)$ correspond with the vertices in $\iota_{i}(\mathbb{T}(\mathcal{L}))$.

A chain in a graph $\mathbb{G}$ is a sub-graph of $\mathbb{G}$ formed by a sequence $\left[v_{1}, v_{2}, \ldots, v_{n}\right]$ of vertices such that for $1 \leq i \leq n-1, v_{i}$ is connected to $v_{i+1}$ by an edge $e_{i}$. We call a chain proper if all its vertices are distinct. A loop in a graph $\mathbb{G}$ is a chain $\left[v_{1}, v_{2}, \ldots, v_{n}\right]$ with $v_{1}=v_{n}$. If $v_{1}$ and $v_{2}$ are vertices of a tree $\mathbb{T}, \zeta\left(v_{1}, v_{2}\right)$ denotes the unique proper chain connecting $v_{1}$ to $v_{2}$.

Example 1.1. For the finite measured lamination $\mathcal{L}$ shown in Figure 1, we choose the external complementary component $E_{1}$ to correspond with the vertex $v(1)$ in $\partial \mathbb{T}(\mathcal{L})$. Then $\iota_{3}(\mathbb{T}(\mathcal{L}))$ consists of vertices corresponding with the components $E_{4}$, $I_{2}, I_{1}$, and $E_{2}$.

\section{Grafting And simultaneous Bending}

Finite grafting. A lens of angle $\theta$ is a Möbius image of the closure of the region $\left\{z \in \mathbb{C}: z=r e^{i \vartheta}, r>0,0<\vartheta<\theta\right\}$ in $\mathbf{S}^{2}$. Suppose $l$ is a geodesic in $\mathbf{D}$ with endpoints $e_{1}$ and $e_{2}$ and $\mathcal{M}$ is the elliptic Möbius map with fixed points at $e_{1}$ and $e_{2}$ and rotation angle $\theta$ in the clockwise direction as viewed from $e_{1}$. $\mathbf{D} \backslash l$ has two components; $\mathcal{A}_{1}$ which is to the left of $l$ when we move from $e_{1}$ to $e_{2}$ and the other component which we denote by $\mathcal{A}_{2}$. A simple grafting by angle $\theta$ along a geodesic $l$ is defined as follows. We cut along $l$ and then we glue a lens of angle $\theta$ so that for any point on $l$ the corresponding points $a_{1}$ and $a_{2}$ on the boundaries of $\mathcal{A}_{1}$ and $\mathcal{A}_{2}$ after the gluing satisfy $a_{2}=\mathcal{M}\left(a_{1}\right)$. This defines a new Möbius (projective) structure on the disk which is denoted by $\mathcal{G}_{\theta l}(\mathbf{D})$. For definitions regarding Möbius structures see [11] and [19]. Given a finite measured lamination $\mathcal{L}$ on $\mathbf{D}$ with weights $\theta_{i}$ on leaves $l_{i}, 1 \leq i \leq n$, we define $\mathcal{G}_{\mathcal{L}}(\mathbf{D})$ to be the Möbius (projective) structure on the disk which is obtained from the standard Möbius structure on $\mathbf{D}$ by doing simple graftings $\mathcal{G}_{\theta_{i} l_{i}}(\mathbf{D}), 1 \leq i \leq n$ (see Figure 22). For any component $\mathcal{A}_{i}$ of $\mathbf{D} \backslash \mathcal{L}$ there is a natural Möbius map $f_{i}: \mathcal{A}_{i} \rightarrow \mathcal{G}_{\mathcal{L}}(\mathbf{D})$ which extends to $\partial \mathcal{A}_{i}$. One can piece together all these extension maps on $\partial \mathcal{A}_{i} \cap \mathbf{S}^{1}$ to define a continuous map $\mathcal{G}_{\mathcal{L}}: \mathbf{S}^{1} \rightarrow \partial \mathcal{G}_{\mathcal{L}}(\mathbf{D}) . \mathcal{G}_{\mathcal{L}}$ is defined only up to post-composition with a Möbius map.

Realizable pairs of finite measured laminations. Suppose $\mathcal{L}^{+}$and $\mathcal{L}^{-}$are finite measured laminations. Let $E^{\sigma}, \sigma= \pm$ be the set of the endpoints of the 

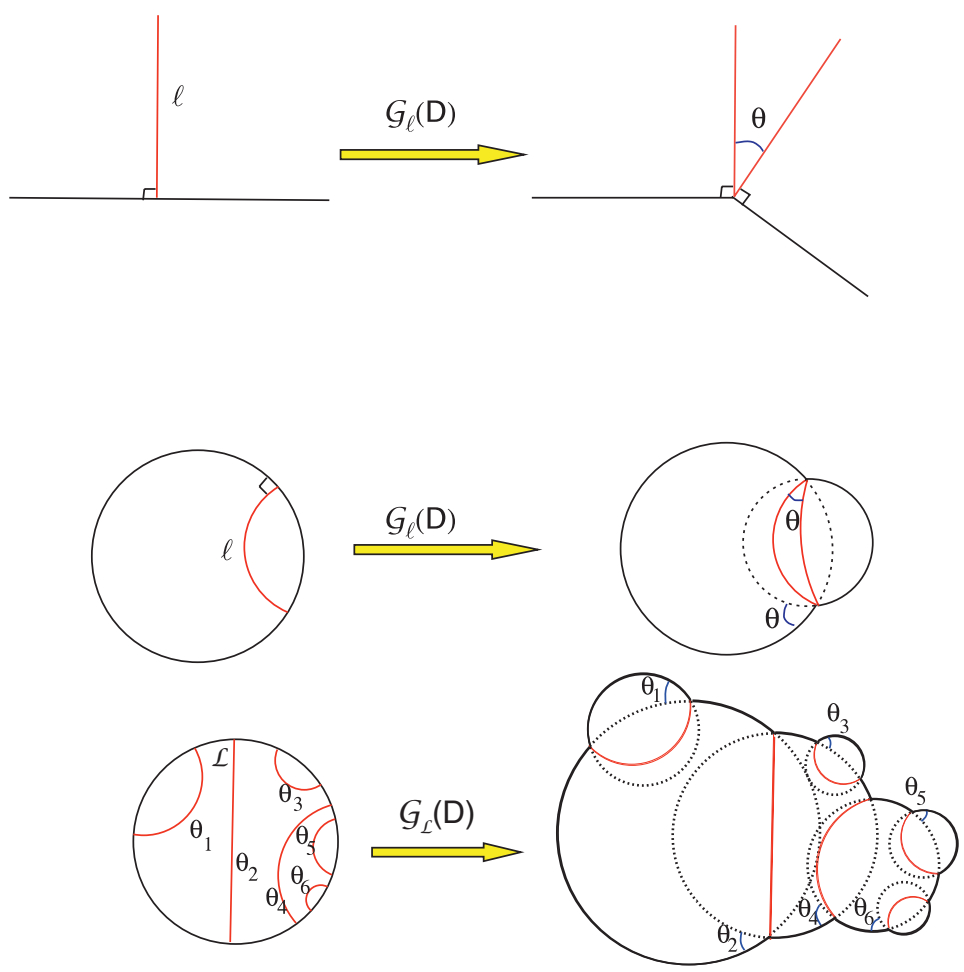

FiguRE 2.

leaves of $\mathcal{L}^{\sigma}$. Moreover, assume no two different leaves share an endpoint. In particular, $E^{+} \cap E^{-}=\varnothing$. The sign $\sigma$ is called the type of the leaf or lamination or an endpoint.

Definition 2.1. Suppose $\left(\mathcal{L}^{+}, \mathcal{L}^{-}\right)$is a pair of finite measured laminations with sets of endpoints $E^{+}$and $E^{-}$in $\mathbf{S}^{1}$ and denote $E^{+} \cup E^{-}$by $E .\left(\mathcal{L}^{+}, \mathcal{L}^{-}\right)$is called realizable if there are maps $f^{+}: E \rightarrow \mathbf{S}^{1}$ and $f^{-}: E \rightarrow \mathbf{S}^{1}$, preserving the cyclic order of points, such that:

(1) The extensions of the developing maps of $\mathcal{G}_{f^{+}\left(\mathcal{L}^{+}\right)}(\mathbf{D})$ and $\mathcal{G}_{f^{-}\left(\mathcal{L}^{-}\right)^{*}}\left(\mathbf{D}^{*}\right)$ to $\overline{\mathbf{D}}$ and $\overline{\mathbf{D}}^{*}$ define homeomorphisms $\mathcal{G}^{+}: \mathbf{S}^{1} \rightarrow \partial \mathcal{G}_{f^{+}(\mathcal{L}+)}(\mathbf{D})$ and $\mathcal{G}^{-}$: $\mathbf{S}^{1} \rightarrow \partial \mathcal{G}_{f^{-}\left(\mathcal{L}^{-}\right)^{*}}\left(\mathbf{D}^{*}\right)$.

(2) There is an order preserving Möbius map $F$ such that $\mathcal{G}_{f^{+}\left(\mathcal{L}^{+}\right)}\left(f^{+}(x)\right)=$ $F\left(\mathcal{G}_{f^{-}\left(\mathcal{L}^{-}\right)^{*}}\left(f^{-}(x)\right)\right)$ for all $x \in E=E^{+} \cup E^{-}$.

The pair $\left(f^{+}, f^{-}\right)$is called a simultaneous realization marking pair for $\left(\mathcal{L}^{+}, \mathcal{L}^{-}\right)$and the set $\mathcal{G}_{f^{+}\left(\mathcal{L}^{+}\right)}\left(f^{+}(E)\right)$ (as well as $\left.\mathcal{G}_{f^{-}\left(\mathcal{L}^{-}\right)^{*}}\left(f^{-}(E)\right)\right)$ up to application of a Möbius map is called a simultaneous realization of $\left(\mathcal{L}^{+}, \mathcal{L}^{-}\right)$. If $\left(f^{+}, f^{-}\right)$is a simultaneous realization marking pair for $\left(\mathcal{L}^{+}, \mathcal{L}^{-}\right)$, then for any Möbius maps $M^{+}$and $M^{-}$preserving $\mathbf{S}^{1},\left(M^{+} \circ f^{+}, M^{-} \circ f^{-}\right)$is also a simultaneous realization marking pair for $\left(\mathcal{L}^{+}, \mathcal{L}^{-}\right)$.

Let $\left(\mathcal{L}_{1}^{+}, \mathcal{L}_{1}^{-}\right)$and $\left(\mathcal{L}_{2}^{+}, \mathcal{L}_{2}^{-}\right)$be two pairs of finite measured laminations with sets of endpoints $E_{1}$ and $E_{2}$. We say $\left(\mathcal{L}_{1}^{+}, \mathcal{L}_{1}^{-}\right)$and $\left(\mathcal{L}_{2}^{+}, \mathcal{L}_{2}^{-}\right)$are equivalent if there is a bijection $h: E_{1} \rightarrow E_{2}$ preserving the cyclic order of points such that 


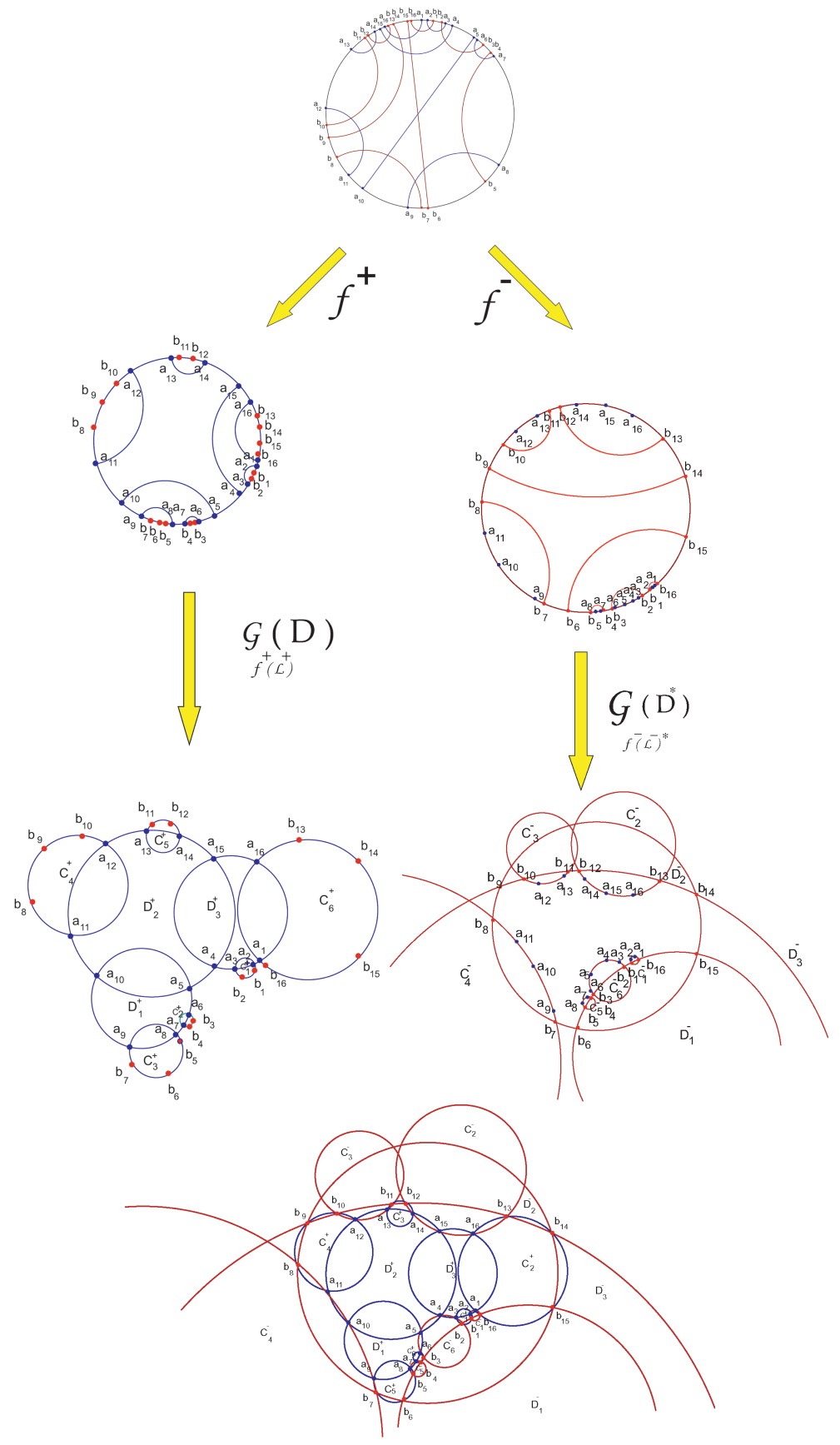

Figure 3 . Set $E$ of the endpoints after grafting along laminations $\mathcal{L}^{+}$and $-\mathcal{L}^{-}$.

$h\left(\mathcal{L}_{1}^{+}\right)=\mathcal{L}_{2}^{+}$and $h\left(\mathcal{L}_{1}^{-}\right)=\mathcal{L}_{2}^{-}$. It is clear from the definition that a pair of finite measured laminations that is equivalent to a realizable pair, is also realizable, and two equivalent realizable pairs admit the same simultaneous realizations. 

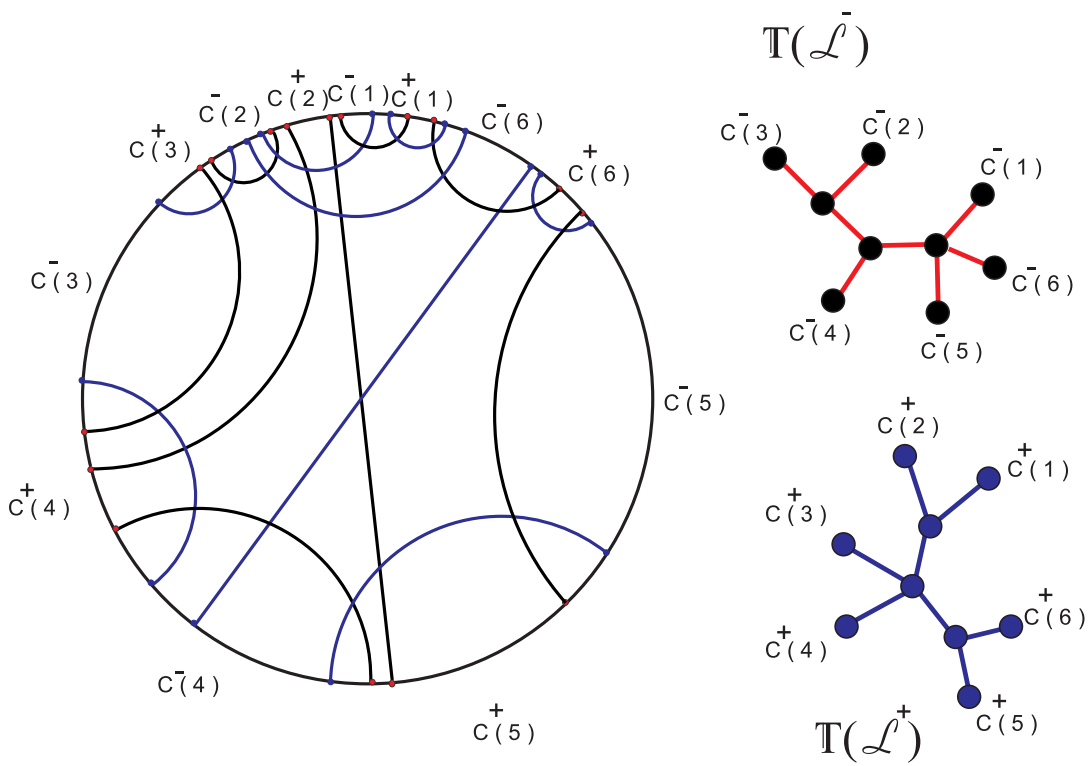

Figure 4. A fully binding pair $\left(\mathcal{L}^{+}, \mathcal{L}^{-}\right)$of finite laminations and the corresponding weighted planar rooted trees

Definition 2.2. A pair $\left(\mathcal{L}^{+}, \mathcal{L}^{-}\right)$of finite laminations is called fully binding if every external leaf of each lamination intersects two distinct external leaves of the other lamination.

The main objective of this paper is to prove the following theorem:

Theorem 2.3 (Finite simultaneous bending). Suppose $\left(\mathcal{L}^{+}, \mathcal{L}^{-}\right)$is a fully binding pair of finite measured laminations and all leaves are assigned the same weight $0<\vartheta<\pi$. Moreover, assume that no two leaves share an endpoint. Then $\left(\mathcal{L}^{+}, \mathcal{L}^{-}\right)$ is realizable.

Proof. The theorem follows from theorem 4.16 and lemma 3.5.

Remark 2.4. In example 4.4 we will show why, in theorem 2.3. we should assume all the weights are equal to the same number.

For the pair $\left(\mathcal{L}^{+}, \mathcal{L}^{-}\right)$to be realizable it is not necessary to be fully binding. Here we define a weaker notion that is a necessary condition.

Definition 2.5. A pair $\left(\mathcal{L}^{+}, \mathcal{L}^{-}\right)$of finite laminations is called binding if for any component $\mathcal{A}$ of $\mathbf{D} \backslash\left(\mathcal{L}^{+} \cup \mathcal{L}^{-}\right), \partial \mathcal{A} \cap \mathbf{S}^{1}$ is connected.

Notice that an external leaf of each lamination can intersect at most two distinct external leaves of the other lamination. When a pair is fully binding every external leaf intersects exactly two external leaves of the other lamination and therefore a neighborhood of $\mathbf{S}^{1}$ is covered by external components of the two laminations. This means the closure of any component $\mathcal{A}$ of $\mathbf{D} \backslash\left(\mathcal{L}^{+} \cup \mathcal{L}^{-}\right)$is either compact or $\mathcal{A}$ is inside an external component of one of the two laminations. Moreover, in the second case, $\mathcal{A} \cap \mathbf{S}^{1}$ is a connected interval. Therefore, fully binding pairs are binding as well. 
The following lemma shows that being binding is necessary for being realizable.

Lemma 2.6. Any realizable pair of finite measured laminations is binding.

Proof. Suppose for a connected component $\mathcal{A}$ of $\mathbf{D} \backslash\left(\mathcal{L}^{+} \cup \mathcal{L}^{-}\right), \partial \mathcal{A} \cap \mathbf{S}^{1}$ is not connected and therefore contains at least two disjoint intervals $I_{1}$ and $I_{2}$ on $\mathbf{S}^{1}$. For $i=1,2$, let $a_{i}$ and $b_{i}$ be the endpoints of $I_{i}$. These four points are in the endpoint set $E=E^{+} \cup E^{-}$. Denote the connected component of $\mathbf{D} \backslash \mathcal{L}^{\sigma}$ that contains $\mathcal{A}$ by $\mathcal{A}^{\sigma}$ and let $F$ be the Möbius map in the definition of realizable pairs. Since $a_{1}$, $b_{1}, a_{2}$, and $b_{2}$ all lie on $\partial \mathcal{A}^{\sigma} \cap \mathbf{S}^{1}$, on $\partial \mathcal{G}_{f^{+}\left(\mathcal{L}^{+}\right)}(\mathbf{D})$ and $\partial F\left(\mathcal{G}_{f^{-}\left(\mathcal{L}^{-}\right)^{*}}\left(\mathbf{D}^{*}\right)\right)$ they must lie on boundaries of disks $D^{+}$and $D^{-}$such that we have $D^{+} \subset \mathcal{G}_{f^{+}(\mathcal{L}+)}(\mathbf{D})$ and $D^{-} \subset F\left(\mathcal{G}_{f^{-}\left(\mathcal{L}^{-}\right)^{*}}\left(\mathbf{D}^{*}\right)\right) . \partial D^{-}$and $\partial D^{+}$share four points. Therefore, they must coincide and since $F$ is orientation preserving, $D^{-}$must be the closure of the complement of $D^{+}$. Hence for any point $x \in E^{+} \cup E^{-}$that is not on $\partial \mathcal{A}^{\sigma} \cap \mathbf{S}^{1}$, $\mathcal{G}_{f^{+}\left(\mathcal{L}^{+}\right)}\left(f^{+}(x)\right)$ must be outside $D^{+}$and $F\left(\mathcal{G}_{f^{-}\left(\mathcal{L}^{-}\right)^{*}}\left(f^{-}(x)\right)\right)$ must be outside $D^{-}$. This is a contradiction unless $\mathcal{G}_{f+\left(\mathcal{L}^{+}\right)}(\mathbf{D})=D^{+}$and $F\left(\mathcal{G}_{f^{-}\left(\mathcal{L}^{-}\right)^{*}}\left(\mathbf{D}^{*}\right)\right)=D^{-}$or, equivalently, if both $\mathcal{L}^{+}$and $\mathcal{L}^{-}$are empty.

In example 4.17 we will show that there are binding pairs of finite laminations which are realizable but are not fully binding.

In order to prove theorem 2.3 we first show that it is equivalent to the existence of a configuration of disks parameterized by pairs of weighted planar rooted trees. This will be done in the next section.

\section{Projective COMPlexes OF DISKS}

Given a fully binding pair of finite laminations, the external components cover a neighborhood of $\partial \mathbf{D}=\mathbf{S}^{1}$. Labeling one external component of type + as $\mathfrak{C}^{+}(1)$, we get a cyclic labeling of all external components by going around $\mathbf{S}^{1}$ in the counterclockwise direction. Right after $\mathcal{C}^{+}(1)$ there is an external component of type - which is labeled $\mathrm{C}^{-}(1)$ and then $\mathrm{C}^{+}(2)$ appears until we get back to $\mathrm{C}^{+}(1)$ as we move around $\mathbf{S}^{1}$ a full turn. Notice that this labeling is compatible with the labeling of the vertices in $\partial \mathbb{T}\left(\mathcal{L}^{+}\right)$and $\partial \mathbb{T}\left(\mathcal{L}^{-}\right)$described in section 1 . We also observe that $\partial \mathbb{T}\left(\mathcal{L}^{+}\right)$and $\partial \mathbb{T}\left(\mathcal{L}^{-}\right)$contain the same number $N$ of vertices. Let $\mathcal{A}$ be a connected component of $\mathbf{D} \backslash \mathcal{L}^{\sigma}$. Then the number of the connected components of $\partial \mathcal{A} \cap \mathbf{S}^{1}$ is equal to the valence of the vertex of $\mathbb{T}\left(\mathcal{L}^{\sigma}\right)$ corresponding with $\mathcal{A}$. Each connected component of $\partial \mathcal{A} \cap \mathbf{S}^{1}$ corresponds with one of the $\iota_{i}\left(\mathbb{T}\left(\mathcal{L}^{\sigma}\right)\right)$ 's that contain the vertex of $\mathbb{T}\left(\mathcal{L}^{\sigma}\right)$ corresponding with $\mathcal{A}$. For $i \in \mathbb{Z}_{N}$, if $\sigma=+$, the connected component of $\partial \mathcal{A} \cap \mathbf{S}^{1}$ corresponding with $\iota_{i}\left(\mathbb{T}\left(\mathcal{L}^{+}\right)\right)$lies inside $\partial \mathcal{C}^{-}(i) \cap \mathbf{S}^{1}$ and if $\sigma=-$, the connected component of $\partial \mathcal{A} \cap \mathbf{S}^{1}$ corresponding with $\iota_{i}\left(\mathbb{T}\left(\mathcal{L}^{-}\right)\right)$ lies inside $\partial \mathcal{C}^{+}(i+1) \cap \mathbf{S}^{1}$ (see Figure 4).

$\mathbb{T}\left(\mathcal{L}^{\sigma}\right)$ by itself determines $\mathcal{L}^{\sigma}$ up to application of an order preserving map $h^{\sigma}: E^{\sigma} \rightarrow \mathbf{S}^{1}$. Moreover, as a result of the above paragraph, by choosing $\mathcal{C}^{-}(1)$ to be the external component of $\mathcal{L}^{-}$that appears right after $\mathcal{C}^{+}(1)$, as we turn around $\mathbf{S}^{1}$ in the counterclockwise direction, we can also encode the way $\mathcal{L}^{+}$and $\mathcal{L}^{-}$are entangled with each other. Therefore, the pair $\left(\mathbb{T}\left(\mathcal{L}^{+}\right), \mathbb{T}\left(\mathcal{L}^{-}\right)\right)$of planar weighted rooted trees captures the same data as the equivalence class of the fully binding pair $\left(\mathcal{L}^{+}, \mathcal{L}^{-}\right)$. For this reason, from now on, we will consider pairs of rooted planar weighted trees instead of fully binding pairs of finite measured laminations. 
We fix our notation by the following definitions:

Definition 3.1. For a pair $\left(D_{1}, D_{2}\right)$ of disks, there are three possibilities:

- The two disks intersect in a lens of angle $\phi$. In this case we call the pair of disks space-like and we denote $\pi-\phi$ by $\theta\left(D_{1} D_{2}\right)$.

- $\partial D_{1} \cap \partial D_{2}=\varnothing$. In this case we call the pair of disks time-like and we denote the translation length of the hyperbolic (nonloxodromic) Möbius map that maps $\partial D_{1}$ to $\partial D_{2}$ by $d\left(D_{1} D_{2}\right)$.

- $\partial D_{1} \cap \partial D_{2}$ consists of one point. In this case we call the pair of disks light-like.

Definition 3.2. We call a triple $D_{0} D_{1} D_{2}$ of disks ideal if $\partial D_{0} \cap \partial D_{1} \cap \partial D_{2}$ consists of exactly one point.

As it can be seen in Figure 3, simultaneous realization of a pair $\left(\mathcal{L}^{+}, \mathcal{L}^{-}\right)$of finite measured laminations, defines a configuration of disks with a combinatorial pattern prescribed by $\left(\mathbb{T}\left(\mathcal{L}^{+}\right), \mathbb{T}\left(\mathcal{L}^{-}\right)\right)$. We call this configuration of disks a complex of disks. Complexes of disks are parameterized by pairs $\left(\mathbb{T}^{+}, v^{+}(1)\right),\left(\mathbb{T}^{-}, v^{-}(1)\right)$ of weighted planar rooted trees. The formal definition is given below.

Definition 3.3. Suppose $\left(\left(\mathbb{T}^{+}, v^{+}(1)\right),\left(\mathbb{T}^{-}, v^{-}(1)\right)\right)$ is a pair of weighted planar rooted trees with $\#\left(\partial \mathbb{T}^{+}\right)=\#\left(\partial \mathbb{T}^{-}\right)=N$ such that all the weights $w$ satisfy $0<w<\pi$. A complex of disks parameterized by $\left(\left(\mathbb{T}^{+}, v^{+}(1)\right),\left(\mathbb{T}^{-}, v^{-}(1)\right)\right)$ is a finite collection of closed disks $\left\{D_{i}^{+}\right\} \cup\left\{D_{j}^{-}\right\}$such that:

(1) The disks $D_{i}^{\sigma}$ correspond with the vertices of $\mathbb{T}^{\sigma}$. If $D_{k}^{\sigma}$ and $D_{l}^{\sigma}$ are connected by an edge of weight $w$, then $D_{k}^{\sigma}$ and $D_{l}^{\sigma}$ form a space-like pair with $\theta\left(D_{k}^{\sigma} D_{l}^{\sigma}\right)=w$. We denote the external disk corresponding with the external vertex $v(i) \in \partial \mathbb{T}^{\sigma}$ by $C^{\sigma}(i)$. Disks $D^{\sigma}$ are called internal (respectively external) disks of type $\sigma$ if they correspond to vertices of $\operatorname{int}\left(\mathbb{T}^{\sigma}\right)$ (respectively $\partial\left(\mathbb{T}^{\sigma}\right)$ ).

(2) For any external disk $C^{+}(i)$ (respectively $C^{-}(i)$ ) and any disk $D^{-} \in$ $\iota_{i-1}\left(\mathbb{T}^{-}\right)$which is not $C^{-}(i)$ (respectively any disk $D^{+} \in \iota_{i}\left(\mathbb{T}^{+}\right)$which is not $\left.C^{+}(i+1)\right), C^{+}(i), D^{-}$, and $s_{i-1}\left(D^{-}\right)$(respectively $C^{-}(i), D^{+}$, and $\left.s_{i}\left(D^{+}\right)\right)$form an ideal triple.

(3) Let $D$ be a vertex of $\mathrm{T}^{\sigma}$ with valence $m$ and suppose $D^{i}, i \in \mathbb{Z}_{m}$ are the vertices in $j(D)$ labeled in the clockwise cyclic order these vertices appear around $D$. Let $I_{i}=\partial D \cap D^{i}$. Then $I_{i} \cap I_{j}=\varnothing$ when $i \neq j$. Moreover, $I_{i}$ 's appear on $\partial D$ in the clockwise direction in the same order they are labeled when $\sigma=+$ (respectively in the opposite order when $\sigma=-$ ). (See Figure 5)

(4) For $\left.k \neq l, \sigma= \pm, \overline{\left(\partial D_{k}^{\sigma} \backslash \bigcup_{D_{i}^{\sigma} \in j\left(D_{k}^{\sigma}\right)} D_{i}^{\sigma}\right)} \cap \overline{\left(\partial D_{l}^{\sigma} \backslash \bigcup_{D_{j}^{\sigma} \in j\left(D_{l}^{\sigma}\right)} D_{j}^{\sigma}\right.}\right)=\varnothing$. See remark 3.4 below for additional comments on this condition.

Remark 3.4. Condition (4) in the definition of complexes of disks implies that the union of the disks of the same type forms a simply connected region on the sphere. See the middle of Figure 6 where the union of the disks is not simply connected and as a result it fails to satisfy condition (4). On the other hand, condition (4) without condition (3) is stronger than the simple connectivity of the union of the disks of the same type. The left-hand side of Figure 6 shows an example of the disks of type + with the simply connected union failing to satisfy conditions (3) and 

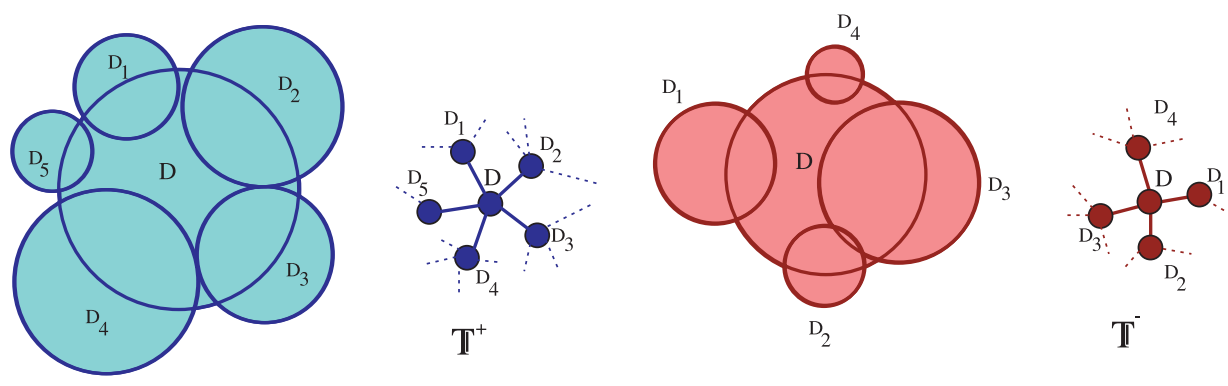

FiguRE 5 .

(4). The right-hand side of Figure 6 shows the disks of type + satisfying condition (4). As it can be seen, this does not prevent the disks that are not connected by edges to intersect each other. It turns out that conditions (3) and (4) are what we need to show that the union $U^{\sigma}$ of the disks of each type is obtained from the grafting of the unit disk along a finite measured lamination and the extension of the developing map of $U^{\sigma}$ to $\overline{\mathbf{D}}$ defines a homeomorphism from $\mathbf{S}^{1}$ to $\partial U^{\sigma}$.
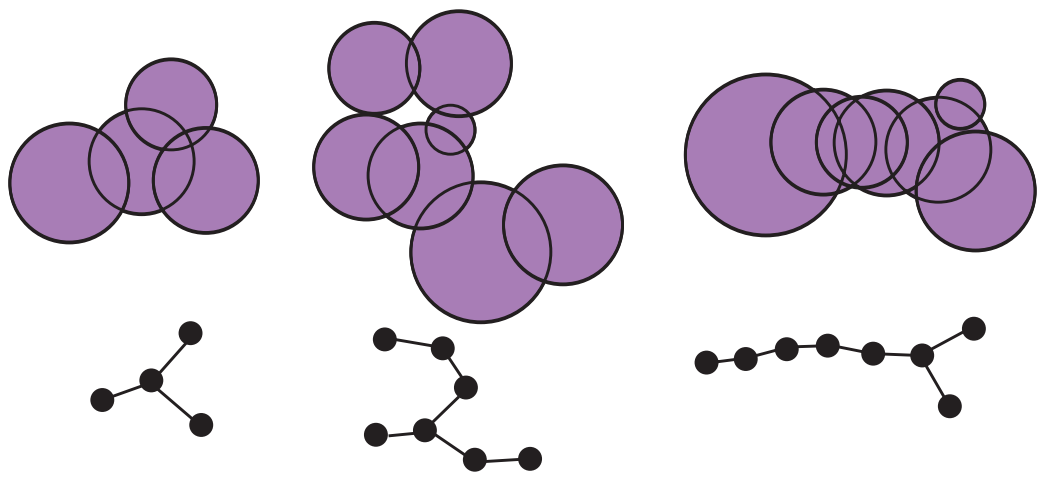

FiguRE 6.

The following lemma clarifies the relation between the finite simultaneous bending and complexes of disks.

Lemma 3.5. Suppose a fully binding pair $\left(\mathcal{L}^{+}, \mathcal{L}^{-}\right)$of finite measured laminations is realizable. Then any realization of $\left(\mathcal{L}^{+}, \mathcal{L}^{-}\right)$can be described as the set of all points of intersection between the boundaries of external disks with the boundaries of disks of opposite type in a complex of disks with parameters $\mathbb{T}\left(\mathcal{L}^{\sigma}\right), \sigma= \pm$. Conversely, any complex of disks with parameters $\mathbb{T}\left(\mathcal{L}^{\sigma}\right), \sigma= \pm$ defines a simultaneous realization of $\left(\mathcal{L}^{+}, \mathcal{L}^{-}\right)$.

Proof. Let $\mathcal{A}$ be a complementary component of $\mathcal{L}^{\sigma}$. $\mathcal{A}$ is a piecewise circular region in $\mathbf{S}^{2}$. The circular arcs forming $\partial \mathcal{A}$ alternate between leaves of $\mathcal{L}^{\sigma}$ and $\operatorname{arcs}$ $\gamma_{i}$ on the unit circle. These arcs connect endpoints of type $\sigma$ and there are some endpoints of opposite type lying on $\gamma_{i}$. Since grafting of $\mathbf{D}$ along $f^{+}\left(\mathcal{L}^{+}\right)$(respectively grafting of $\mathbf{D}^{*}$ along $\left.f^{-}\left(\mathcal{L}^{-}\right)^{*}\right)$ does not change the configuration of the set of endpoints of $\mathcal{L}^{-}$(respectively $\mathcal{L}^{+}$) on $\partial \mathcal{A} \cap \mathbf{S}^{1}$ up to the application of Möbius 
maps, on $\partial \mathcal{G}_{f^{+}\left(\mathcal{L}^{+}\right)}(\mathbf{D})$ (respectively $\partial \mathcal{G}_{f^{-}\left(\mathcal{L}^{-}\right)^{*}}\left(\mathbf{D}^{*}\right)$ ), these endpoints still lie on the boundary of a disk. We denote this disk by $D_{\mathcal{A}}$. Let $F$ be the Möbius map in the definition of realizable pairs of finite measured laminations. We claim that the union of the disks $D_{\mathcal{A}}$ for all complementary components $\mathcal{A}$ of $\mathcal{L}^{+}$together with the disks $F\left(D_{\mathcal{B}}\right)$ for all complementary components $\mathcal{B}$ of $\mathcal{L}^{-}$form a complex of disks with parameters $\mathbb{T}\left(\mathcal{L}^{\sigma}\right), \sigma= \pm$ (see Figure 3 ). If $\mathcal{A}$ and $\mathcal{B}$ are two complementary components of $\mathcal{L}^{\sigma}$ that share a leaf of $\mathcal{L}^{\sigma}$ with weight $w$ on their boundaries, then $D_{\mathcal{A}}^{\sigma}$ and $D_{\mathcal{B}}^{\sigma}$ will intersect each other in a lens of angle $\pi-w$. Condition (3) of complexes of disks follows from the definition of grafting. If condition (4) of complexes of disks is not satisfied, there must be a pair $\mathcal{A}_{1}$ and $\mathcal{A}_{2}$ of complementary components of $\mathcal{L}^{\sigma}$ for which $\partial D_{\mathcal{A}_{1}} \backslash \bigcup_{D_{i} \in j\left(D_{\mathcal{A}_{1}}\right)} D_{i}$ intersects $\partial D_{\mathcal{A}_{2}} \backslash \bigcup_{D_{j} \in j\left(D_{\mathcal{A}_{2}}\right)} D_{j}$. Notice that $\partial \mathcal{G}_{f^{+}\left(\mathcal{L}^{+}\right)}(\mathbf{D})$ (respectively $\partial \mathcal{G}_{f^{-}\left(\mathcal{L}^{-}\right)^{*}}\left(\mathbf{D}^{*}\right)$ ) is formed of closed circular arcs in $\overline{\partial D_{\mathcal{A}} \backslash \bigcup_{D_{i} \in j\left(D_{\mathcal{A}}\right)} D_{i}}$ for complementary components $\mathcal{A}$ of $\mathcal{L}^{+}$(respectively $\mathcal{L}^{-}$) and any such arc appears on the boundary of exactly one $D_{\mathcal{A}}$. Therefore existence of a point in the intersection of $\overline{\partial D_{\mathcal{A}_{1}} \backslash \bigcup_{D_{i} \in j\left(D_{\mathcal{A}_{1}}\right)} D_{i}}$ and $\overline{\partial D_{\mathcal{A}_{2}} \backslash \bigcup_{D_{j} \in j\left(D_{\mathcal{A}_{2}}\right)} D_{j}}$ for some pair $\mathcal{A}_{1}$ and $\mathcal{A}_{2}$ is equivalent to $\partial \mathcal{G}_{f^{+}\left(\mathcal{L}^{+}\right)}(\mathbf{D})\left(\right.$ respectively $\partial \mathcal{G}_{f^{-}\left(\mathcal{L}^{-}\right)^{*}}\left(\mathbf{D}^{*}\right)$ ) having a self-intersection which is against the first condition in the definition of realizable pairs of finite measured laminations. Condition (2) of complexes of disks is satisfied by condition (2) in the definition of realizable pairs. Moreover, the points in the given realization of $\left(\mathcal{L}^{+}, \mathcal{L}^{-}\right)$are exactly the union of the points of intersection of the boundaries of $C^{+}(i), D^{-}$, and $s_{i-1}\left(D^{-}\right)$for $C^{+}(i) \in \partial \mathbb{T}\left(\mathcal{L}^{+}\right)$and $D^{-} \in \iota_{i-1}\left(\mathbb{T}^{-}\right)$which is not $C^{-}(i)$ and the points of intersection of the boundaries of $C^{-}(i), D^{+}$, and $s_{i}\left(D^{+}\right)$for $C^{-}(i) \in \partial \mathbb{T}\left(\mathcal{L}^{-}\right)$and $D^{+} \in \iota_{i}\left(\mathbb{T}^{+}\right)$which is not $C^{+}(i+1)$.

Conversely, let $\mathcal{Q}$ be a complex of disks with parameters $\mathbb{T}\left(\mathcal{L}^{\sigma}\right), \sigma= \pm$. By property (3) of complexes of disks, for any disk $D$ of type $\sigma$ and distinct disks $D_{1}$ and $D_{2}$ in $j(D), \partial D \cap D_{1}$ and $\partial D \cap D_{2}$ are disjoint intervals. Moreover, the intervals $\partial D \cap D^{\prime}$ for $D^{\prime} \in j(D)$ appear around $D$ in the cyclic order prescribed by $\mathbb{T}\left(\mathcal{L}^{\sigma}\right)$. This together with condition (1) in the definition of complexes of disks imply the union of all disks of type + (respectively disks of type -) defines a Möbius structure on the disk which is Möbius equivalent to $\mathcal{G}_{\widetilde{\mathcal{L}^{+}}}(\mathbf{D})$ (respectively $\mathcal{G}_{\widetilde{\mathcal{L}^{-}}}\left(\mathbf{D}^{*}\right)$ ) for some finite measured lamination $\widetilde{\mathcal{L}^{\sigma}}$ satisfying $\mathbb{T}(\widetilde{\mathcal{L} \sigma})=\mathbb{T}\left(\mathcal{L}^{\sigma}\right)$. As we saw above, condition (4) of complexes of disks implies that there are homeomorphisms $h_{+}$: $\mathbf{S}^{1} \rightarrow \partial \mathcal{G}_{\widetilde{\mathcal{L}^{+}}}(\mathbf{D})$ and $h_{-}: \mathbf{S}^{1} \rightarrow \partial \mathcal{G}_{\widetilde{\mathcal{L}^{-}}}\left(\mathbf{D}^{*}\right)$.

Let $\mathcal{E}^{\sigma}$ be the set of all points that appear in the boundary intersections of pairs of adjacent disks of type $\sigma$ and denote $\mathcal{E}^{+} \cup \mathcal{E}^{-}$by $\mathcal{E}$. Moreover, following the comments in the beginning of this section and condition 2 in the definition of complexes of disks, since the points in $h_{\sigma}^{-1}(\mathcal{E})$ appear in the cyclic order prescribed by the pair $\left(\mathbb{T}(\widetilde{\mathcal{L}+}), \mathbb{T}\left(\widetilde{\mathcal{L}^{-}}\right)\right)=\left(\mathbb{T}\left(\mathcal{L}^{+}\right), \mathbb{T}\left(\mathcal{L}^{-}\right)\right)$, there is a map $f^{\sigma}$ from $E$ onto $h_{\sigma}{ }^{-1}(\mathcal{E})$ which preserves the cyclic order of points on $\mathbf{S}^{1}$. Furthermore, $\left\{\mathcal{G}_{f^{+}\left(\mathcal{L}^{+}\right)}\left(f^{+}(x)\right) \mid x \in E\right\}$ is Möbius equivalent to $\left\{\mathcal{G}_{f^{-}\left(\mathcal{L}^{-}\right)^{*}}\left(f^{-}(x)\right) \mid x \in E^{-}\right\}$. Hence, $\left(\mathcal{L}^{+}, \mathcal{L}^{-}\right)$is realizable.

\section{THE INDUCED TRIANGULATION AND DISK PATTERNS}

Complexes of disks can be regarded as disk patterns parameterized by a weighted triangulation of the sphere. Each disk corresponds with a vertex of the 
triangulation and the weight on each edge $e$ determines the angle of the intersection between the disks represented by the vertices joined by $e$. The triangulation (2-dimensional simplicial complex) that parameterizes the disk pattern in a complex of disks, however, highlights a pair of disjoint trees that define the triangulation in the following way.
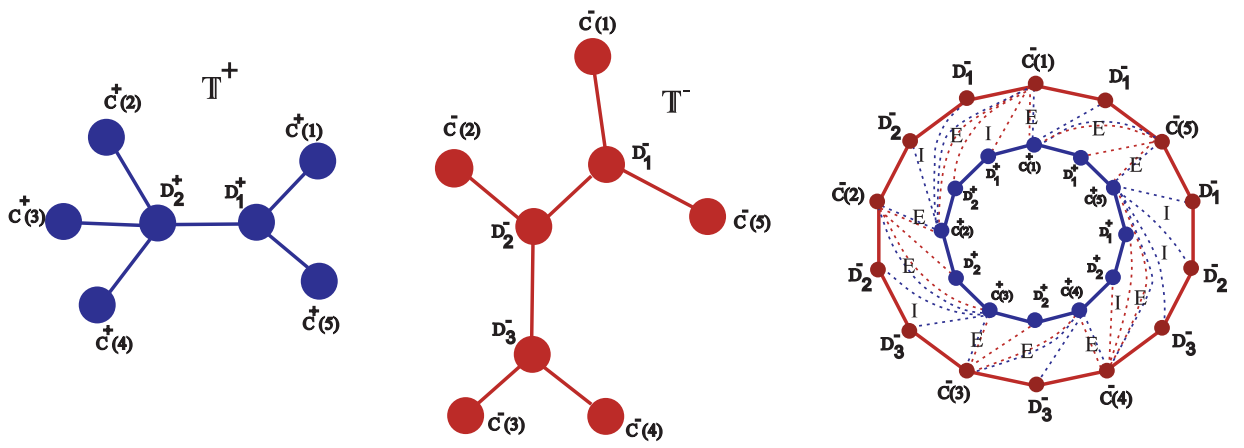

Figure 7. Left: A pair of planar trees $\mathbb{T}^{\sigma}, \sigma= \pm$. Right: $\mathcal{C}^{\sigma}\left(\mathbb{T}^{\sigma}\right)$, $\sigma= \pm$. Red dotted lines connect the vertices $C^{-}(i) \in \Pi^{-1}\left(\partial \mathbb{T}^{-}\right)$to vertices in $\Pi^{-1}\left(\iota_{i}\left(\mathbb{T}^{+}\right)\right)$and blue dotted lines connect the vertices $C^{+}(i) \in \Pi^{-1}\left(\partial \mathbb{T}^{+}\right)$to vertices in $\Pi^{-1}\left(\iota_{i-1}\left(\mathbb{T}^{-}\right)\right)$. Any two adjacent dotted lines together with an edge of $e^{\sigma}\left(\mathbb{T}^{\sigma}\right)$ form a face of $T\left(\mathbb{T}^{+}, \mathbb{T}^{-}\right)$.

Definition 4.1. Let $\left(\mathbb{T}^{+}, \mathbb{T}^{-}\right)$be a pair of planar rooted trees with $\# \partial \mathbb{T}^{+}=$ $\# \partial \mathbb{T}^{-}=N$. We define the triangulation $T\left(\mathbb{T}^{+}, \mathbb{T}^{-}\right)$of $\mathbf{S}^{2}$ as follows (see Figure 8 ):

- The vertices of $T\left(\mathbb{T}^{+}, \mathbb{T}^{-}\right)$are formed of the union of the vertices of $\mathbb{T}^{+}$ and $\mathbb{T}^{-}$.

- The edges of $T\left(\mathbb{T}^{+}, \mathbb{T}^{-}\right)$are formed of the union of the edges of $\mathbb{T}^{+}$and $\mathbb{T}^{-}$together with the edges in the forms $C^{+}(i) D^{-}, C^{+}(i) \in \partial \mathbb{T}^{+}, D^{-} \in$ $\iota_{i-1}\left(\mathbb{T}^{-}\right)$and $C^{-}(i) D^{+}, C^{-}(i) \in \partial \mathbb{T}^{-}, D^{+} \in \iota_{i}\left(\mathbb{T}^{+}\right)$.

- Faces of $T\left(\mathbb{T}^{+}, \mathbb{T}^{-}\right)$are either in the form $C^{+}(i) D^{-} s_{i-1}\left(D^{-}\right), C^{+}(i) \in \partial \mathbb{T}^{+}$, $D^{-} \in \iota_{i-1}\left(\mathbb{T}^{-}\right), D^{-} \neq C^{-}(i)$, or are in the form $C^{-}(i) D^{+} s_{i}\left(D^{+}\right), C^{-}(i) \in$ $\partial \mathbb{T}^{-}, D^{+} \in \iota_{i}\left(\mathbb{T}^{-}\right), D^{+} \neq C^{+}(i+1)$.

In order to see that the above combinatorial data in fact defines a triangulation of $\mathbf{S}^{2}$, notice that by considering the loops $\mathcal{C}\left(\mathbb{T}^{+}\right)$and $\mathcal{C}\left(\mathbb{T}^{-}\right)$and joining the pairs of vertices $C^{+}(i) D^{-}, C^{+}(i) \in \partial \mathbb{T}^{+}, D^{-} \in \iota_{i-1}\left(\mathbb{T}^{-}\right)$and $C^{-}(i) D^{+}, C^{-}(i) \in \partial \mathbb{T}^{-}$, $D^{+} \in \iota_{i}\left(\mathbb{T}^{-}\right.$), we obtain a triangulation of an annulus (see Figure 7 ). Then we glue back along the edges of $\mathcal{C}\left(\mathbb{T}^{ \pm}\right)$to get the triangulation $T\left(\mathbb{T}^{+}, \mathbb{T}^{-}\right)$of the sphere.

As it can be seen in Figure 8, the embedding of $T\left(\mathbb{T}^{+}, \mathbb{T}^{-}\right)$on the sphere forces one of the two planar trees to appear as its mirror image. To be compatible with the third condition of complexes of disks, we choose to flip $\mathbb{T}^{-}$and keep $\mathbb{T}^{+}$unchanged.

When $\left(\mathcal{L}^{+}, \mathcal{L}^{-}\right)$is a fully binding pair of finite geodesic laminations, the 1skeleton of $T\left(\mathbb{T}\left(\mathcal{L}^{+}\right), \mathbb{T}\left(\mathcal{L}^{-}\right)\right)$can be defined in the following way. Every vertex corresponds with a connected component of either $\mathbf{D} \backslash \mathcal{L}^{+}$or $\mathbf{D}^{*} \backslash\left(\mathcal{L}^{-}\right)^{*}$. Two vertices are connected by an edge if the corresponding regions share a leaf or a subset of $\mathbf{S}^{1}$ on their boundaries. 

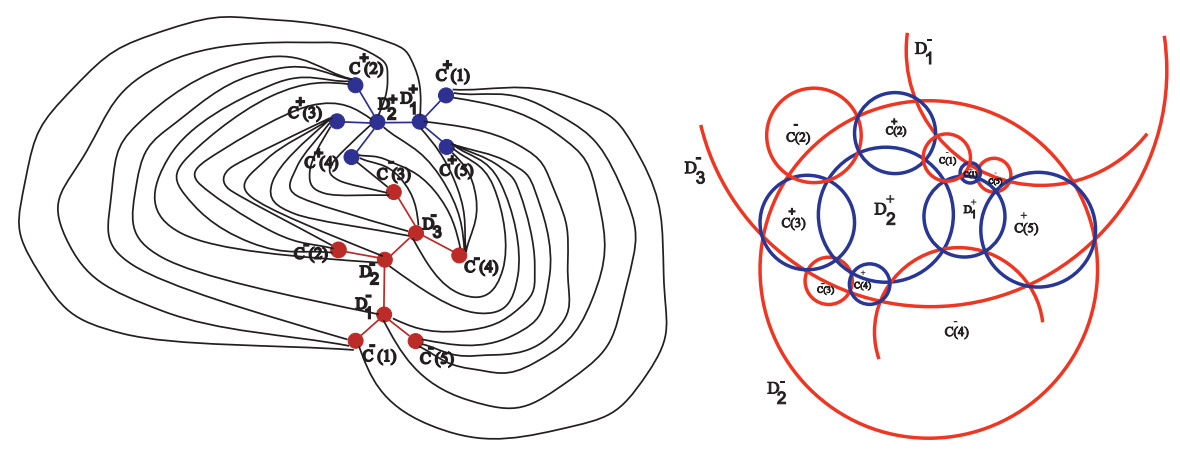

Figure 8. Left: The induced triangulation $T\left(\mathbb{T}^{+}, \mathbb{T}^{-}\right)$for the pair of trees in Figure 7 Right: A complex of disks with parameter trees as in Figure 7

Definition 4.2. Given a triangulation $\mathrm{T}$ of the sphere with an assignment of weights $0<W(e)<\pi$ to each edge $e$, we say a collection $\mathcal{Q}$ of disks is a disk pattern parameterized by $\mathrm{T}$ if there is a bijection $v$ from the disks in $Q$ to the vertices of T such that if $v(D)$ and $v\left(D^{\prime}\right)$ are connected by an edge $e$, then the pair $\left(D, D^{\prime}\right)$ is space-like and $\theta\left(D D^{\prime}\right)=W(e)$. Given a disk pattern, we call a pair $D_{1} D_{2}$ of disks an edge if $v\left(D_{1}\right) v\left(D_{2}\right)$ is an edge of the parameter triangulation $\mathrm{T}$ and we call a triple $D_{1} D_{2} D_{3}$ a face if $v\left(D_{1}\right) v\left(D_{2}\right) v\left(D_{3}\right)$ is a face of the parameter triangulation T. Disk pattern is called ideal if faces $D_{1} D_{2} D_{3}$ of T are ideal triples of disks.

Let $\left(\mathbb{T}^{+}, \mathbb{T}^{-}\right)$be a pair of weighted rooted planar trees with $\#\left(\mathbb{T}^{+}\right)=\#\left(\mathbb{T}^{-}\right)$. If a complex $\mathcal{Q}$ of disks is parameterized by $\left(\mathbb{T}^{+}, \mathbb{T}^{-}\right)$, we can assign weights $W(e)$ to each edge $e$ of $T\left(\mathbb{T}^{+}, \mathbb{T}^{-}\right)$such that the complex $Q$ is a disk pattern parameterized by $T\left(\mathbb{T}^{+}, \mathbb{T}^{-}\right)$. For any edge $D_{1} D_{2}$ of $\mathbb{T}^{\sigma}$ with a weight $w(e)$ assigned to it in $\mathbb{T}^{\sigma}$, by the first condition in the definition of complexes of disks, we have $W(e)=$ $\theta\left(D_{1} D_{2}\right)=w(e)$. The second condition in the definition of complexes of disks states that every face $D_{1} D_{2} D_{3}$ of $T\left(\mathbb{T}^{+}, \mathbb{T}^{-}\right)$is an ideal triple. Notice that for a triple $D_{1} D_{2} D_{3}$ of pairwise space-like disks, the triple is ideal exactly when $\theta\left(D_{1} D_{2}\right)+$ $\theta\left(D_{2} D_{3}\right)+\theta\left(D_{1} D_{3}\right)=2 \pi$. Once we choose a weight $0<W<\pi$ for any edge of $T\left(\mathbb{T}^{+}, \mathbb{T}^{-}\right)$that is not an edge of $\mathbb{T}^{ \pm}$, the weights on the rest of such edges are forced by the above observation that the sum of the weights assigned to the edges of each face must be equal to $2 \pi$. The forced weights may, however, be outside the interval $(0, \pi)$. It is also possible that by choosing an arbitrary weight $W$ on an edge $e$ of $T\left(\mathbb{T}^{+}, \mathbb{T}^{-}\right)$that is not an edge of $\mathbb{T}^{ \pm}$, and following all the forced assignments of weights, we end up with a weight other than $W$ on $e$ (see example 4.4 below). This gives a necessary condition for a pair of rooted planar weighted trees to be parameter trees for a complex of disks.

Definition 4.3. We say an assignment of weights $w(l)$ to the edges of the pair of trees $\left(\mathbb{T}^{+}, \mathbb{T}^{-}\right)$is compatible if there is an assignment $W(e)$ of weights to the edges $e$ of the triangulation $T\left(\mathbb{T}^{+}, \mathbb{T}^{-}\right)$such that:

- All the weights $W(e)$ are in the interval $(0, \pi)$.

- $W(e)=w(e)$, for any edge $e$ of $\mathbb{T}^{ \pm}$.

- The sum of the weights assigned to the edges of each face is equal to $2 \pi$. 
We say an assignment of weights $w(l)$ to the leaves of a pair $\left(\mathcal{L}^{+}, \mathcal{L}^{-}\right)$of finite geodesic laminations is compatible if the induced assignment of weights on the edges of the pair of trees $\left(\mathbb{T}\left(\mathcal{L}^{+}\right), \mathbb{T}\left(\mathcal{L}^{-}\right)\right)$is compatible.
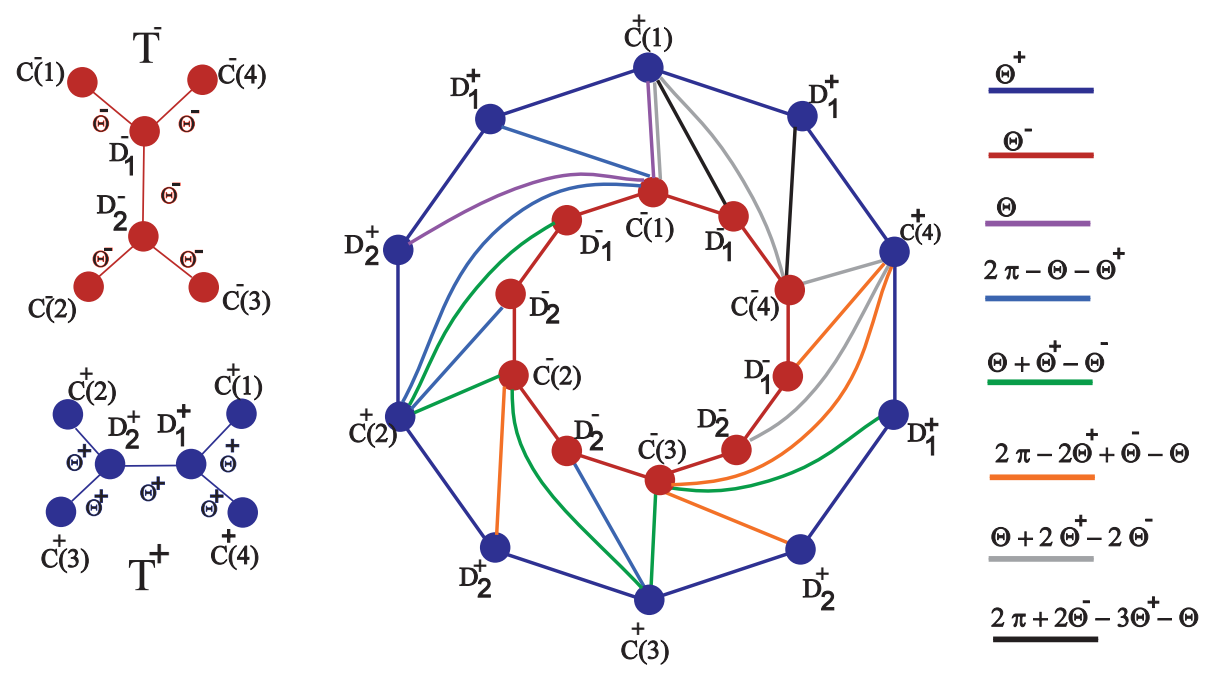

Figure 9.

Example 4.4. We consider the pair of trees shown on the left side of Figure 9 , We assign the same weight $\Theta^{\sigma}$ to all edges of $\mathbb{T}^{\sigma}$. By assigning the weight $\Theta$ on the edge $C^{-}(1) C^{+}(1)$ the rest of the weights are determined and it is shown on the right-hand side of Figure 9 that by going around once we come back to $C^{-}(1) C^{+}(1)$ with assignment of the weight $\Theta+2 \Theta^{+}-2 \Theta^{-}$which is not equal to $\Theta$ if $\Theta^{+} \neq \Theta^{-}$. This example shows that compatibility is not an open condition on the space of assignments of weights to the edges of a fixed pair of rooted planar trees.

Assignment of the same number $0<\vartheta<\pi$ to all the leaves of $\mathcal{L}^{ \pm}$is compatible. It is because the weights of the edges of $\mathbb{T}\left(\mathcal{L}^{ \pm}\right)$in $T\left(\mathbb{T}\left(\mathcal{L}^{+}\right), \mathbb{T}\left(\mathcal{L}^{-}\right)\right)$are $\vartheta$ and assignment of $\pi-\frac{\vartheta}{2}$ to the rest of the edges satisfies the conditions in the definition of compatibility. Each face of $T\left(\mathbb{T}^{+}, \mathbb{T}^{-}\right)$has one edge of the trees $\mathbb{T}^{ \pm}$together with two edges that are assigned the weight $\pi-\frac{\vartheta}{2}$. Therefore, the sum of the weights on the edges of each face is $2 \pi$.

Definition 4.5. A complex of disks is called homogeneous if all the weights on its parameter trees are equal to the same number $0<\vartheta<\pi$ and for any edge $D D^{\prime}$ of $T\left(\mathbb{T}\left(\mathcal{L}^{+}\right), \mathbb{T}\left(\mathcal{L}^{-}\right)\right)$that is not an edge of $\mathbb{T}^{ \pm}, \theta\left(D D^{\prime}\right)=\pi-\frac{\vartheta}{2}$. Notice that if for one such edge we have $\theta\left(D D^{\prime}\right)=\pi-\frac{\vartheta}{2}$, then all other such edges are also forced to satisfy the same property.

The third condition in the definition of complexes of disks implies that the corresponding disk pattern is maximal.

Definition 4.6. A disk pattern $Q$ parameterized by a weighted triangulation $\mathrm{T}$ is called maximal if, for any pair of disks $D_{1}$ and $D_{2}$ that are connected by edges $D D_{1}$ and $D D_{2}$ to $D$, the interiors of $\partial D \cap D_{1}$ and $\partial D \cap D_{2}$ do not intersect. 
The term maximal is used in [5] for the unbranched disk patterns following the analogous term for circle packings. The first three conditions in definition 3.3 are equivalent to the existence of a maximal ideal disk pattern parameterized by $T\left(\mathbb{T}^{+}, \mathbb{T}^{-}\right)$. In what follows, we will show how the fourth condition in definition 3.3 follows from the other conditions and homogeneity of the complex. When no weight is smaller than $\frac{\pi}{2}$, the pairs of disks for which the corresponding vertices are not connected, do not intersect (see for example [9] and it also follows from lemma 4.8). Since we are interested in assignments of weights that are in the interval $(0, \pi)$, it is important to understand which pairs of disks may intersect.

We equip $\mathbf{S}^{2}$ with a metric of constant positive curvature and denote the center of each disk $D$ with respect to the spherical metric by $c(D)$. For any pair $D_{1} D_{2}$ of disks with nonempty intersection, let $g\left(D_{1}, D_{2}\right)$ be the unique geodesic segment that joins $c\left(D_{1}\right)$ to $c\left(D_{2}\right)$ and is a subset of $D_{1} \cup D_{2}$. For a face $D_{1} D_{2} D_{3}, \mathcal{T}\left(D_{1}, D_{2}, D_{3}\right)$ is the spherical triangle with sides $g(A, B), g(B, C)$, and $g(C, A)$. Suppose we are given a maximal ideal disk pattern $\mathcal{Q}$ parameterized by a triangulation $\mathrm{T}$. We define a map $f_{\mathcal{Q}}$ from the abstract triangulation $\mathrm{T}$ to the sphere as follows. $f_{\mathcal{Q}}$ maps any vertex $D$ of $\mathrm{T}$ to $c(D)$ and it maps any edge $D_{1} D_{2}$ to $g\left(D_{1}, D_{2}\right)$. Then we extend $f_{\mathcal{Q}}$ from the 1 -skeleton of $\mathrm{T}$ to $\mathrm{T}$ so that any face $D_{1} D_{2} D_{3}$ is mapped homeomorphically to $\mathcal{T}\left(D_{1}, D_{2}, D_{3}\right)$. $f_{\mathrm{Q}}$ is a local homeomorphism inside the faces and on the edges. Let $D_{i}, i \in \mathbb{Z}_{N}$ be the vertices connected to $D$ labeled in the order they appear. Since the great circle that contains $g\left(D, D_{i}\right)$ passes through $D \cap D_{i}$, maximality implies that $g\left(D, D_{i}\right)$ appear in the cyclic order they are labeled and therefore $\mathcal{T}\left(D, D_{i}, D_{i+1}\right), i \in \mathbb{Z}_{N}$, form a neighborhood of $c(D)$ so that $f_{\mathcal{Q}}$ is also a local homeomorphism at the vertex $D$. Since $f_{\mathcal{Q}}$ is a local homeomorphism of the sphere, it must be a homeomorphism.

Lemma 4.7. Suppose $Q$ is a maximal ideal disk pattern parameterized by a triangulation $\mathrm{T}$ of the sphere. Then:

(a) If $D$ and $D^{\prime}$ are disks in $Q$, then $D \nsubseteq D^{\prime}$.

(b) If $D, D_{1}$, and $D_{2}$ are are disks in $Q$, and $D_{1}$ and $D_{2}$ are connected, then $D \nsubseteq D_{1} \cup D_{2}$.

Proof. (a) We consider the map $f_{Q}$ constructed above. Using a Möbius map, we may assume $c(D)=c\left(D^{\prime}\right)$. Although $f_{\mathcal{Q}}$ depends on $\mathcal{Q}$, its being a homeomorphism is true for any Möbius image of $\mathcal{Q}$ as well. On the other hand, $c(D)=c\left(D^{\prime}\right)$ implies $f_{Q}(D)=f_{Q}\left(D^{\prime}\right)$ which contradicts injectivity of $f_{Q}$.

(b) We can apply a Möbius map so that $c\left(D^{\prime}\right) \in g\left(D_{1}, D_{2}\right)$ which contradicts injectivity of $f_{\mathcal{Q}}$ as it means $f_{\mathcal{Q}}\left(D^{\prime}\right) \in f_{\mathcal{Q}}\left(D_{1} D_{2}\right)$.

As a result of lemma 4.7, it is not possible for disks $D$ and $D^{\prime}$ in the given disk pattern to satisfy $D \subset D^{\prime}$. Therefore, if the interiors of $D$ and $D^{\prime}$ intersect, they must be space-like. We try to understand the intersection of pairs of disks in maximal ideal disk patterns in the following lemma.

Lemma 4.8. Suppose $Q$ is a maximal ideal disk pattern parameterized by a triangulation $\mathrm{T}$ of the sphere. If the disks $D$ and $D^{\prime}$ intersect but they are not connected by an edge of $\mathrm{T}$, then there is a unique proper chain $\left[D_{1}=D, D_{2}, \ldots, D_{n}=D^{\prime}\right]$ such that $D \cap D^{\prime} \subset D_{i}, 1 \leq i \leq n$. 
Proof. There is a sequence $\mathcal{T}_{i}, 1 \leq i \leq m$, of triangles (see Figure 10, left) such that:

- $\mathcal{T}_{i}=\mathfrak{T}\left(A_{i}, B_{i}, C_{i}\right)$ for a face $A_{i} B_{i} C_{i}$.

- For $1 \leq i \leq m-1, \mathcal{T}_{i}$ and $\mathcal{T}_{i+1}$ either share a side or they share a vertex.

- $D$ is a vertex of $\mathcal{T}_{1}$ and $D^{\prime}$ is a vertex of $\mathcal{T}_{m}$.

- $g\left(D, D^{\prime}\right)$ is covered by $\mathcal{T}_{i}$ 's and the segments $\mathcal{T}_{i} \cap g\left(D, D^{\prime}\right)$ appear in the order $\mathcal{T}_{i}$ 's are labeled.
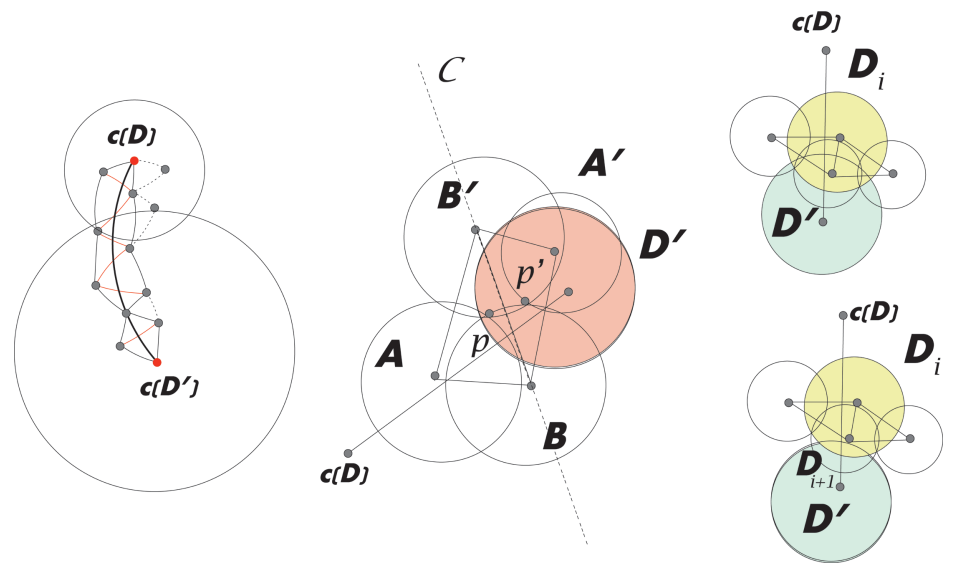

Figure 10.

We make the following observations:

(a) Suppose that $\mathcal{T}_{i}=\mathcal{T}\left(A, B, B^{\prime}\right)$ and $\mathcal{T}_{i+1}=\mathcal{T}\left(B, B^{\prime}, A^{\prime}\right)$ for faces $A B B^{\prime}$ and $B B^{\prime} A^{\prime}$. Let $p$ and $p^{\prime}$, respectively, be the points of intersection $\partial A \cap \partial B \cap \partial B^{\prime}$ and $\partial A^{\prime} \cap \partial B \cap \partial B^{\prime}$. Then if $D^{\prime}$ contains $p$, it must also contain $p^{\prime}$ (see Figure 10, middle). This can be shown in the following way. Let $\mathcal{C}$ be the great circle on the sphere that contains $g\left(B, B^{\prime}\right)$. $\mathcal{C}$ is the locus of the points that are equidistant from $p$ and $p^{\prime}$. Since $g\left(D, D^{\prime}\right)$ should intersect at least two sides of $\mathcal{T}_{i+1}, c\left(D^{\prime}\right)$ must lie in the complementary component of $\mathcal{C}$ that contains $c\left(A^{\prime}\right)$. Then the spherical distance between $c\left(D^{\prime}\right)$ and $p$ is more than the spherical distance between $c\left(D^{\prime}\right)$ and $p^{\prime}$ and therefore $D^{\prime}$ contains $p^{\prime}$.

(b) We assume that for all $1 \leq i \leq m-1, \mathcal{T}_{i}$ and $\mathcal{T}_{i+1}$ share an edge. Let $\mathcal{T}_{m}=\mathcal{T}\left(D^{\prime}, A_{m}, B_{m}\right)$. Then there is no index $1 \leq i \leq m-1$, such that $\mathcal{T}_{i}=\mathcal{T}\left(A_{i}, B_{i}, C_{i}\right)$ and $\left\{p_{i}\right\}=\partial A_{i} \cap \partial B_{i} \cap \partial C_{i}$ is contained in $D^{\prime}$. It is because existence of such $\mathcal{T}_{i}$, by a repeated use of part (a), results in the point $p_{m}=\partial D^{\prime} \cap A_{m} \cap B_{m}$ being inside $D^{\prime}$ which is a contradiction.

(c) We still assume that for all $1 \leq i \leq m-1, \mathcal{T}_{i}$ and $\mathcal{T}_{i+1}$ share an edge. $D^{\prime} \cap \partial D$ is covered by a chain $\left[C_{1}, C_{2}, \ldots, C_{l}\right]$ of disks that are connected to $D$ in T. If $l>1$, then there is an index $1 \leq i \leq l-1$ such that $\mathcal{T}_{1}=\mathcal{T}\left(D C_{i} C_{i+1}\right)$ and therefore $D^{\prime}$ must contain $\partial D \cap \partial C_{i} \cap \partial C_{i+1}$ which by part (b) is impossible. Therefore, there is a disk $D_{2}$ connected to $D$ such that $\partial D \cap D^{\prime} \subset D_{2}$. Let $\tilde{D}$ be the disk that passes through the points in $\partial D \cap \partial D_{2}$ and $\theta(\tilde{D} D)=\theta\left(D^{\prime} D\right)$ (see Figure 11, right). Since $\partial D \cap D^{\prime} \subset D_{2}$, $D^{\prime} \subset \tilde{D}$. If $D \cap D^{\prime} \nsubseteq D_{2} \cap D$, we must have $\theta(\tilde{D} D)=\theta\left(D^{\prime} D\right) \geq \theta\left(D D_{2}\right)$ 
which implies $D^{\prime} \subset \tilde{D} \subset D \cup D_{2}$ and, by lemma 4.7, is impossible. Hence $D \cap D^{\prime} \subset D_{2} \cap D$. Moreover, $g\left(D, D^{\prime}\right)$ intersects $D_{2} \cap D$.

Now there is a chain of disks connected to $D_{2}$ that cover $\partial D_{2} \cap D^{\prime}$. If this chain contains more than one disk, the centers of two of them together with $c\left(D_{2}\right)$ must form a triangle $\mathcal{T}_{i}$ and the point of intersection of the boundaries of the three disks must be inside $D^{\prime}$ (see Figure 10, right). Again, this is ruled out by (b). Continuing this argument we obtain a chain $\left[D_{1}=\right.$ $\left.D, D_{2}, \ldots, D_{n}=D^{\prime}\right]$ such that for any $1 \leq i \leq n-1, D^{\prime} \cap D_{i} \subset D^{\prime} \cap D_{i+1}$. As a result $D^{\prime} \cap D \subset D^{\prime} \cap D_{i}$, for $1<i<n$. Hence all $D_{i}$ 's contain $D \cap D^{\prime}$. Notice that the chain $\left[D_{1}=D, D_{2}, \ldots, D_{n}=D^{\prime}\right]$ is uniquely determined by the property $D \cap D^{\prime} \subset D_{i}, 1<i<n$, because as we observed above, for each $1<i<n$, among all the disks connected to $D_{i}$ only one has this property.

(d) If for some $1 \leq i \leq m, \mathcal{T}_{i}$ and $\mathcal{T}_{i+1}$ share a vertex $v$ but not an edge, then $g\left(D, D^{\prime}\right)$ must pass through $v$. We prove the general case by induction on the number $M$ of vertices of $\mathcal{T}_{i}$ 's that lie on $g\left(D, D^{\prime}\right)$. The case $M=2$ with only $c(D)$ and $c\left(D^{\prime}\right)$ being on $g\left(D, D^{\prime}\right)$ was proven in (c). Let $M>2$ and suppose $c\left(D^{\prime \prime}\right)$ lies on $g\left(D, D^{\prime}\right)$ and $D^{\prime \prime}$ is distinct from $D$ and $D^{\prime}$. Then by induction hypothesis, there are unique proper chains $\left[D_{1}=D, \ldots, D_{k}=\right.$ $\left.D^{\prime \prime}\right]$ and $\left[D_{k}=D^{\prime \prime}, \ldots, D_{n}=D^{\prime}\right]$ that satisfy $D \cap D^{\prime \prime} \subset D_{i}$, for $1 \leq i \leq k$ and $D^{\prime} \cap D^{\prime \prime} \subset D_{i}$, for $k \leq i \leq n$. Notice that since $c\left(D^{\prime \prime}\right)$ is on $g\left(D, D^{\prime}\right)$ and $D^{\prime \prime}$ is not entirely inside $D$ or $D^{\prime}$, if $D^{\prime \prime}$ does not contain $D \cap D^{\prime}$, we must have $\partial D^{\prime \prime} \cap D_{k-1} \cap D_{k+1} \neq \varnothing$ (see Figure 11, left) which is in contradiction to maximality of the disk pattern. Hence $\left[D_{1}=D, \ldots, D_{n}=D\right]$ is the chain of disks claimed by the lemma.
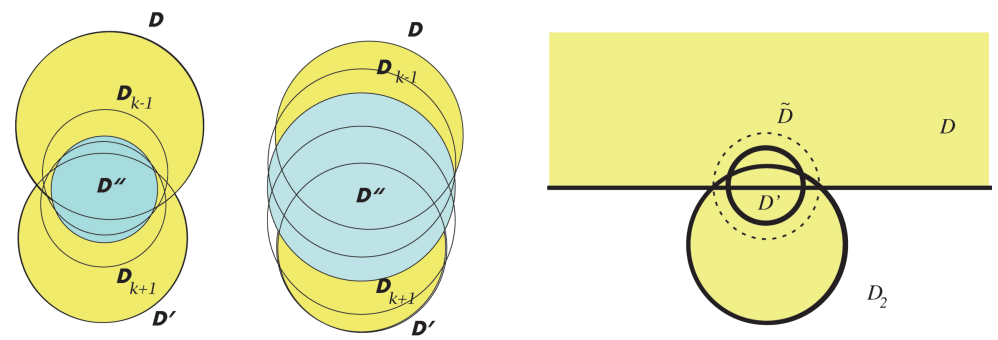

FiguRE 11.

Corollary 4.9. Suppose $\mathcal{Q}$ is a maximal ideal disk pattern parameterized by a triangulation $\mathrm{T}$ of the sphere. Let $\epsilon>0$ be the minimum of the weights assigned to the edges of $T$. Then, if the disks $D$ and $D^{\prime}$ in $Q$ intersect, we must have $\theta\left(D D^{\prime}\right) \geq \epsilon$.

Proof. (See Figure 12). By lemma 4.8, there is a unique proper chain $\left[D_{1}=D\right.$, $\left.D_{2}, \ldots, D_{n}=D^{\prime}\right]$ such that $D \cap D^{\prime} \subset D_{i}, 1 \leq i \leq n$. By uniqueness we also have $D \cap D_{i+1} \subset D_{i}$. Since $D_{i+1} \nsubseteq D_{i}$, the portion of $\partial D_{i+1}$ that is not inside $D$ is not entirely covered by $D_{i}$. Let $\hat{D}$ be the disk that satisfies $\theta(D \hat{D})=\theta\left(D D_{i+1}\right)$ and $\partial \hat{D}$ passes through the two points in $\partial D \cap \partial D_{i}$. $\partial \hat{D}$ is shown by a dotted 


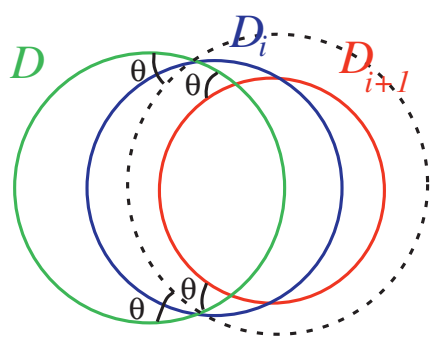

Figure 12.

circle in Figure 12. Since $\partial \hat{D}$ and $\partial D_{i+1}$ intersect $\partial D_{i}$ in the same angle and $\partial D_{i} \cap D_{i+1} \subset \partial D_{i} \cap \hat{D}$ we must have $\partial \hat{D} \cap \partial D_{i+1}=\varnothing$ which implies any disk $\tilde{D}$ with $\theta(D \hat{D}) \geq \theta\left(D D_{i+1}\right)$ such that $\partial D \hat{D}$ passes through the two points in $\partial D \cap \partial D_{i}$, contains the portion of $\partial D_{i+1}$ that is not inside $D$. Therefore $\theta\left(D D_{i+1}\right) \geq \theta\left(D D_{i}\right)$. Using this conclusion repeatedly, we get $\theta\left(D D^{\prime}\right) \geq \theta\left(D D_{2}\right) \geq \epsilon$.

Now, we concentrate on the maximal ideal disk patterns parameterized by $T\left(\mathbb{T}^{+}, \mathbb{T}^{-}\right)$for a pair of planar rooted trees. We assume that the same weight $\vartheta$ is assigned to all the edges of $\mathbb{T}^{ \pm}$and every other edge has weight $\pi-\frac{\vartheta}{2}$. Locally, the intersections of disks that are not connected in $T\left(\mathbb{T}^{+}, \mathbb{T}^{-}\right)$can be understood in the following way:

Lemma 4.10. Suppose we have assigned the same weight $\vartheta$ to all the edges of $\mathbb{T}^{ \pm}$and all other edges of $T\left(\mathbb{T}^{+}, \mathbb{T}^{-}\right)$have weight $\pi-\frac{\vartheta}{2}$. For the disk pattern parameterized by the triangulation $T\left(\mathbb{T}^{+}, \mathbb{T}^{-}\right)$suppose $\left[D_{1}, D_{2}, D_{3}\right]$ is a chain in $T\left(\mathbb{T}^{+}, \mathbb{T}^{-}\right)$such that $D_{1} D_{3}$ is not an edge and $D_{1} \cap D_{2} \cap D_{3} \neq \varnothing$. Then $D_{1}, D_{2}$, and $D_{3}$ are all vertices of the same tree $\mathbb{T}^{\sigma}$.
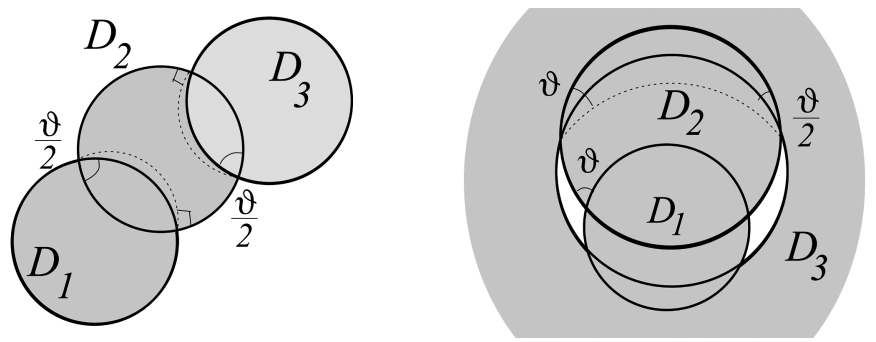

Figure 13.

Proof. If $D_{1}, D_{2}$, and $D_{3}$ are not vertices of the same tree $\mathbb{T}^{\sigma}$, then there are two possibilities:

(a) $D_{1}$ and $D_{3}$ are vertices of the same tree and $D_{2}$ is a vertex of the other tree. In this case $\partial D_{2} \cap D_{1}$ and $\partial D_{2} \cap D_{3}$ are disjoint and $\theta\left(D_{2} D_{1}\right)=$ $\theta\left(D_{2} D_{3}\right)=\pi-\frac{\vartheta}{2}>\frac{\pi}{2}$. Therefore we must have $D_{1} \cap D_{2} \cap D_{3}=\varnothing$ which is a contradiction. (See Figure 13, left.)

(b) Either $D_{1}$ and $D_{2}$ or $D_{3}$ and $D_{2}$ are vertices of the same tree and the other disk is a vertex of the other tree. Without loss of generality we assume $D_{1}$ 
and $D_{2}$ are vertices of the same tree. In this case $\partial D_{2} \cap D_{1}$ and $\partial D_{2} \cap D_{3}$ are disjoint, $\theta\left(D_{2} D_{1}\right)=\vartheta$, and $\theta\left(D_{2} D_{3}\right)=\pi-\frac{\vartheta}{2}$. Since $\frac{\vartheta}{2}<\vartheta, \partial D_{1}$ and $\partial D_{3}$ cannot intersect inside $D_{2}$ and therefore $D_{1} \cap D_{2} \cap D_{3}=\varnothing$ which is a contradiction. (See Figure 13, right.)

Lemma 4.11. Let $\left(\mathbb{T}^{+}, \mathbb{T}^{-}\right)$be a pair of weighted planar rooted trees with internal vertices. Suppose we have assigned the same weight $\vartheta$ to all the edges of $\mathbb{T}^{ \pm}$and all other edges of $T\left(\mathbb{T}^{+}, \mathbb{T}^{-}\right)$have weight $\pi-\frac{\vartheta}{2}$. Suppose that for a disk pattern parameterized by the triangulation $T\left(\mathbb{T}^{+}, \mathbb{T}^{-}\right)$, disks $D$ and $D^{\prime}$ intersect but they are not connected by an edge of $T\left(\mathbb{T}^{+}, \mathbb{T}^{-}\right)$. Then $D$ and $D^{\prime}$ are vertices of the same tree $\mathbb{T}^{\sigma}$ and the unique proper chain $\zeta\left(D, D^{\prime}\right)=\left[D_{1}=D, D_{2}, \ldots, D_{n}=D^{\prime}\right]$ in $\mathbb{T}^{\sigma}$ satisfies $D \cap D^{\prime} \subset D_{i}, 1 \leq i \leq n$.

Proof. By lemma 4.8, there is a unique proper chain $\left[D_{1}=D, D_{2}, \ldots, D_{n}=D^{\prime}\right]$ such that $D \cap D^{\prime} \subset D_{i}, 1 \leq i \leq n$. Since for any $1 \leq i \leq n-2, D \cap D^{\prime} \subset$ $D_{i} \cap D_{i+1} \cap D_{i+2} \neq \varnothing, D_{i} D_{i+1} D_{i+2}$ cannot be an ideal triple and therefore is not a face of $T\left(\mathbb{T}^{+}, \mathbb{T}^{-}\right)$and $D_{i} D_{i+2}$ is not an edge. Therefore, by lemma 4.10, all $D_{i}$ 's must correspond with the vertices of the same tree $\mathbb{T}^{\sigma}$ and since $D_{i}$ 's are distinct and they connect $D$ to $D^{\prime}$ in a tree, the chain $\left[D_{1}=D, D_{2}, \ldots, D_{n}=D^{\prime}\right]$ is uniquely determined as the unique chain $\zeta\left(D, D^{\prime}\right)$ of distinct disks joining $D$ to $D^{\prime}$ in $\mathbb{T}^{\sigma}$.

Among the conditions of complexes of disks, the fourth one is the hardest to check. Here we show that in the homogeneous case, in fact, this condition follows from the other three conditions in definition 3.3 .

Corollary 4.12. Suppose $\left(\mathbb{T}^{+}, \mathbb{T}^{-}\right)$is a pair of planar rooted trees with the assignment of the same weight $0<\vartheta<\pi$ to all its edges. Then, if a configuration of disks satisfies the first three conditions in definition 3.3, and if at least two disks of opposite types that correspond with a pair of vertices joined by an edge in $T\left(\mathbb{T}^{+}, \mathbb{T}^{-}\right)$ intersect each other in a lens of angle $\frac{\vartheta}{2}$, then the configuration of disks must also satisfy the fourth condition in definition 3.3 .

Proof. It follows from the first three conditions in definition 3.3 that the given configuration of disks defines a maximal ideal disk pattern parameterized by $T\left(\mathbb{T}^{+}, \mathbb{T}^{-}\right)$. If for a pair of disks $\left(D, D^{\prime}\right)$ which correspond with the vertices of the same tree $\mathbb{T}^{\sigma}, D \cap D^{\prime} \neq \varnothing$, then by lemma 4.11 for any disk $D_{i}$ in the chain $\zeta\left(D, D^{\prime}\right)=$ $\left[D_{1}=D, D_{2}, \ldots, D_{n}=D^{\prime}\right]$ in $\mathbb{T}^{\sigma}$ we have $D \cap D^{\prime} \subset D_{i}$. Since $D \cap D^{\prime} \subset D_{2}$ and $D_{2} \in j(D)$, we conclude that condition 4 in definition 3.3 is satisfied.

Corollary 4.13. If in a homogeneous complex of disks $D^{+} \in$ int $\mathbb{T}^{+}$and $D^{-} \in$ int $\mathbb{T}^{-}$, then $D^{+} \cap D^{-}=\varnothing$.

Proof. If $D^{+} \cap D^{-} \neq \varnothing$ by lemma 4.11 either $D^{+}$and $D^{-}$must be connected by an edge in $T\left(\mathbb{T}^{+}, \mathbb{T}^{-}\right)$, or they must have the same type. $D^{+}$and $D^{-}$are not connected by an edge in $T\left(\mathbb{T}^{+}, \mathbb{T}^{-}\right)$because they have opposite types and none of them belongs to $\partial \mathbb{T}^{ \pm}$. Therefore, the assumption $D^{+} \cap D^{-} \neq \varnothing$ is ruled out and the corollary follows.

Remark 4.14. Both of the above corollaries are also true when we have assigned compatible weights to the trees $\left(\mathbb{T}^{+}, \mathbb{T}^{-}\right)$and the corresponding disk pattern satisfies the local conclusion of lemma 4.10. This can be guaranteed by knowing that 
whenever there is a chain $\left[D_{1}, D_{2}, D_{3}\right]$ in $T\left(\mathbb{T}^{+}, \mathbb{T}^{-}\right)$such that $D_{1} D_{3}$ is not an edge of $T\left(\mathbb{T}^{+}, \mathbb{T}^{-}\right)$and all three disks are not vertices of the same tree $\mathbb{T}^{\sigma}$, then the sum of the weights assigned to the edges $D_{1} D_{2}$ and $D_{2} D_{3}$ must be greater than $\pi$.

Disk patterns have been studied extensively. The main existence and uniqueness theorem for disk patterns is Andreev's theorem, which assumes that the weights assigned to the edges of the parameter triangulation are all in the interval $\left[\frac{\pi}{2}, \pi\right]$. Since we want to consider the weights that are possibly less than $\frac{\pi}{2}$, we need to apply a more general version of Andreev's theorem proven by Rivin in [14] (see also [15], [16], and [3]).

Theorem 4.15 (Existence and uniqueness of disk patterns). Suppose $\mathrm{T}$ is a triangulation of the sphere and to every edge e of $\mathrm{T}$ we have assigned a weight $W(e)$ such that:

(1) For every edge e, $0<W(e)<\pi$.

(2) The sum of the weights on the three edges of each face is $2 \pi$.

(3) The sum of the weights on the edges of each loop that is not the boundary of a face is strictly greater than $2 \pi$.

Then up to application of Möbius maps, there is a unique maximal ideal disk pattern parameterized by $\mathrm{T}$.

Rivin's theorem is stated for convex ideal hyperbolic polyhedra. A convex polyhedron $\mathcal{P}$ is a finite intersection of half-spaces in the 3 -dimensional hyperbolic space. The boundary of $\mathcal{P}$ inherits a natural cell decomposition and along any edges $e$ of $\mathcal{P}$ one can measure the dihedral angle. A hyperbolic polyhedron is called ideal if all its vertices are on the sphere at infinity. Given a weighted triangulation $\mathrm{T}$, the dual $\mathbf{P}$ of $\mathrm{T}$ is a trivalent abstract weighted polyhedron with the assignment of the weight $\pi-w(e)$ to the edge $e^{*}$ dual to $e$. The form of theorem 4.15 proven by Rivin states that if the weights on $\mathrm{T}$ satisfy the hypothesis of theorem 4.15, then there is a unique convex ideal hyperbolic polyhedron with the cell decomposition structure of $\mathbf{P}$ and dihedral angles prescribed by the weights on the edges of $\mathbf{P}$. For any face $F$ of $\mathcal{P}$, let $H_{F}$ be the half-space that intersects $\mathcal{P}$ in $F$ and let $D_{F}$ be the intersection of the closure of $H_{F}$ with $\mathbf{S}^{2}$. The collection $\mathcal{Q}=\left\{D_{F}: F\right.$ a face of $\mathcal{P}\}$ defines a disk pattern and since $\mathcal{P}$ has the cell decomposition structure of $\mathbf{P}$ and the dihedral angles are prescribed by $\mathbf{P}, Q$ is parameterized by $\mathrm{T}$ and $Q$ is maximal. For any triple $D_{F_{1}} D_{F_{2}} D_{F_{3}}$ of disks in $\mathcal{Q}$ corresponding with a face of $\mathrm{T}$, $F_{1} \cap F_{2} \cap F_{3}$ is a point $v$ on $\mathbf{S}^{2}$ and therefore $\partial D_{F_{1}} \cap \partial D_{F_{2}} \cap \partial D_{F_{3}}=\{v\}$. Hence, $Q$ is ideal as well.

Conversely, suppose we start with an ideal maximal disk pattern $Q$ parameterized by T. For every disk $D \in \mathcal{Q}$ let $D^{c}$ be the closure of the complement of $D$ in $\mathbf{S}^{2}$ and denote the hyperspace whose closure intersects $\mathbf{S}^{2}$ in $D^{c}$ by $H(D)$. The polygon $\mathcal{P}=\bigcap_{D \in \mathcal{Q}} H(D)$, locally around each face, has the cell decomposition structure of $\mathbf{P}$ and the dihedral angles are prescribed by $\mathbf{P}$. By lemma 4.8, any $H\left(D^{\prime}\right)$ for a $D^{\prime}$ that is not adjacent to $D$, contains $H(D) \cap H\left(D_{2}\right)$ for a $D_{2}$ adjacent to $D$. Therefore the local structure of $\mathcal{P}$ around the face defined by $H(D)$ is not changed by intersections with hyperspaces $H\left(D^{\prime}\right)$ for $D^{\prime}$ not adjacent to $D$. As a result $\mathcal{P}$ is realized by $\mathbf{P}$ and therefore Rivin's theorem for polyhedra in fact implies theorem 4.15 for disk patterns.

Knowing Rivin's theorem and corollary 4.12 it is easy to show the existence and uniqueness of complexes of disks. 
Theorem 4.16 (Existence and uniqueness of homogeneous projective complexes). Suppose $\left(\mathbb{T}^{+}, v_{1}^{+}\right)$and $\left(\mathbb{T}^{-}, v_{1}^{-}\right)$are planar rooted trees with weight equal to $0<$ $\vartheta<\pi$ assigned to all their edges. Also assume $\#\left(\partial \mathbb{T}^{+}\right)=\#\left(\partial \mathbb{T}^{-}\right)=N$ and $\operatorname{int}\left(\mathbb{T}^{\sigma}\right) \neq \varnothing$ for $\sigma= \pm$. Then there is a homogeneous projective complex of disks with parameters $\mathbb{T}^{\sigma}, \sigma= \pm$. Moreover, this complex is unique up to projective equivalence.

Proof. By corollary 4.12, the existence and uniqueness of the homogeneous complex parameterized by $\mathbb{T}^{\sigma}, \sigma= \pm$ is equivalent to the existence and uniqueness of a maximal ideal disk pattern parameterized by $T\left(\mathbb{T}^{+}, \mathbb{T}^{-}\right)$. In order to show that such disk pattern exists, and is unique, we should check the hypothesis of theorem 4.15. Obviously, both $\vartheta$ and $\pi-\frac{\vartheta}{2}$ are in the interval $(0, \pi)$. Every face of $T\left(\mathbb{T}^{+}, \mathbb{T}^{-}\right)$ has two edges with weights $\pi-\frac{\vartheta}{2}$ and one edge with weight $\vartheta$. Hence the sum of the weights on the edges of each face is $2 \pi$. Since $\mathbb{T}^{+}$and $\mathbb{T}^{-}$are trees, every loop of $T\left(\mathbb{T}^{+}, \mathbb{T}^{-}\right)$must contain at least two edges that are not edges of $\mathbb{T}^{ \pm}$. Two external disks of opposite types are connected by at most one edge, therefore there are no loops of length two. If a loop of length three does not contain two external disks of the same type, there must be an external disk $C^{\sigma}(i)$ and two disks of opposite type that are connected to each other. Therefore the loop is a boundary of a face. If the loop contains two external disks of the same type $\sigma$, since the third disk is of opposite type, $\mathbb{T}^{\sigma}$ must be two vertices connected by an edge. This is ruled out by the assumption on both trees having internal vertices. Any loop of length greater than three, must contain at least two edges with weight $\pi-\frac{\vartheta}{2}$. If the loop contains an edge of one of the two trees, it contains at least four edges, with sum of the weights on three of them equal to $2 \pi$. There is only one loop that does not contain any edge of $\mathbb{T}^{ \pm}$. This loop is $\left[C^{+}(1), C^{-}(1), C^{+}(2), C^{-}(2), \ldots, C^{+}(N), C^{-}(N), C^{+}(1)\right]$ and the sum of the weights on it is $2 N\left(\pi-\frac{\vartheta}{2}\right)=N(2 \pi-\vartheta)>N \pi \geq 2 \pi$. Therefore, for any loop that is not the boundary of a face, the sum of the weights on the edges of the loop is strictly greater than $2 \pi$.

Example 4.17. The left-hand side of Figure 14 shows a binding pair of finite geodesic laminations which is not fully binding. On the left-hand side, the triangulation $T\left(\mathbb{T}\left(\mathcal{L}^{+}\right), \mathbb{T}\left(\mathcal{L}^{-}\right)\right)$is shown. If we assign the same weight $\vartheta$ to all leaves, then the assignment of the weight $\vartheta$ to all the colored edges and the weight $\pi-\frac{\vartheta}{2}$ to all black edges of $T\left(\mathbb{T}\left(\mathcal{L}^{+}\right), \mathbb{T}\left(\mathcal{L}^{-}\right)\right)$is compatible and it is easy to check that if the sum of the weights on a loop is not greater than $2 \pi$, then the loop is in fact the boundary of a face. Therefore, by Rivin's theorem, there is a disk pattern parameterized by $T\left(\mathbb{T}\left(\mathcal{L}^{+}\right), \mathbb{T}\left(\mathcal{L}^{-}\right)\right)$which, in turn, shows that the pair $\left(\mathcal{L}^{+}, \mathcal{L}^{-}\right)$ is realizable.

Remark 4.18. The homogeneous complex of disks parameterized by a given pair $\left(\mathbb{T}^{+}, \mathbb{T}^{-}\right)$of rooted planar trees may not be the only complex of disks parameterized by $\left(\mathbb{T}^{+}, \mathbb{T}^{-}\right)$. As we also remarked earlier, the assignment of weights to the edges of $T\left(\mathbb{T}^{+}, \mathbb{T}^{-}\right)$, so that edges of the trees are assigned the same weight $\vartheta$ and the sum of the weights on the edges of each face is $2 \pi$, is uniquely determined by the assignment of a weight $\varrho$ to one edge of $T\left(\mathbb{T}^{+}, \mathbb{T}^{-}\right)$which is not an edge of the trees. Then all other such edges will have weights equal to $\varrho$ or $2 \pi-\varrho-\vartheta$. Denote the assignment of weights to $T\left(\mathbb{T}^{+}, \mathbb{T}^{-}\right)$corresponding with a specific value of $\varrho$ by $T_{\varrho}$. It is easy to check that the third condition in theorem 4.15 is satisfied for $T_{\varrho}$ with values of $\varrho$ varying in an interval $I$ containing $\pi-\frac{\vartheta}{2}$. For any such values of $\varrho \in I$, 

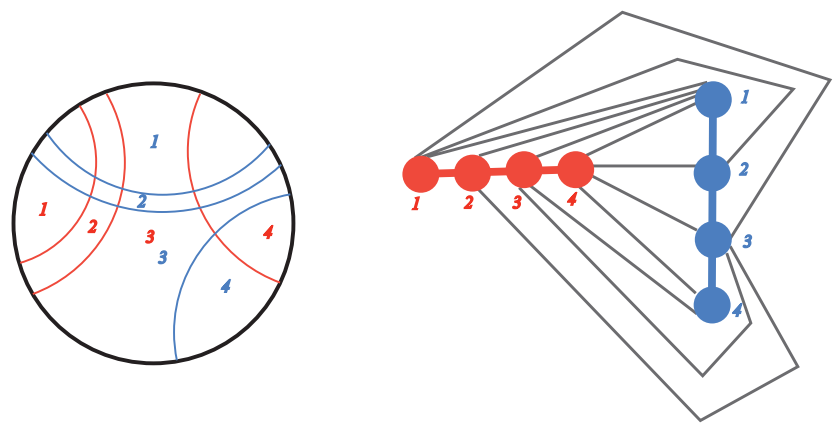

Figure 14.

therefore, there is a unique maximal ideal disk pattern $Q_{\varrho}$ parameterized by $T_{\varrho} . Q_{\varrho}$, $\varrho \in I$, define the only possible configurations of disks, up to application of Möbius maps, satisfying all the conditions in the definition of complexes of disks except possibly for the fourth one. Therefore, there is a family $\left(f_{\varrho}^{+}, f_{\varrho}^{-}\right), \varrho \in I$, of pairs of maps such that any pair $\left(f^{+}, f^{-}\right)$of maps that satisfies the first conclusion of the theorem 2.3 must satisfy $f^{+}=M^{+} \circ f_{\varrho}^{+}$and $f^{-}=M^{-} \circ f_{\varrho}^{-}$, for some $\varrho \in I$ and

Möbius self-maps $M^{+}$and $M^{-}$of $\mathbf{S}^{1}$. Notice that since $2 \pi-\varrho-\vartheta<\pi$ is a weight assigned to some edges of $T_{\varrho}$, we must have $\varrho>\pi-\vartheta$ and therefore $I \subset(\pi-\vartheta, \pi)$.

\section{REFERENCES}

[1] L. Bers, Simultaneous uniformization, Bull. Amer. Math. Soc. 66 (1960), 94-97. MR0111834 $(22: 2694)$

[2] F. Bonahon, Kleinian groups which are almost Fuchsian, J. Reine. Angew. Math. 587 (2005), 1-15. MR 2186972

[3] X. Bao, F. Bonahon, Hyperbolic polyhedra in hyperbolic 3-space, Bull. Soc. Math. France 130 (2002), no. 3, 457-491. MR1943885 (2003k:52007)

[4] F. Bonahon, J.P. Otal, Lamination mesurées de plissage des variétés hyperboliques de dimension 3, Ann. of Math. (2) 160 (2004), no. 3, 1013-1055. MR2144972

[5] P.L. Bowers and K. Stephenson, A branched Andreev-Thurston theorem for circle packings of the sphere, Proc. London Math. Soc. 73 (1996), 185-215. MR1387087 (97d:52027)

[6] Y.E. Choi, C. Series, Lengths are coordinates for convex structures, J. Differential Geometry, to appear.

[7] D.B.A. Epstein, A. Marden, Convex hulls in hyperbolic space, a theorem of Sullivan, and measured pleated surfaces. Analytical and geometric aspects of hyperbolic space (Coventry/Durham, 1984), 113-253, London Math. Soc. Lecture Note Ser., 111, Cambridge Univ. Press, Cambridge, 1987. MR0903852 (89c:52014)

[8] F.P. Gardiner, J. Hu, N. Lakic, Earthquake curves, Complex manifolds and hyperbolic geometry (Guanajuato, 2001), 141-195, Contemp. Math., 311, Amer. Math. Soc., Providence, RI, 2002. MR1940169 (2003i:37033)

[9] Zheng-Xu He, Rigidity of infinite disk patterns, Ann. of Math. (2) 149 (1999), no. 1, 1-33. MR 1680531 (2000j:30068)

[10] C.D. Hodgson, I. Rivin, A characterization of compact convex polyhedra in hyperbolic 3space, Invent. Math. 111 (1993), no. 1, 77-111. MR.1193599 (93j:52015)

[11] R. Kulkarni, U.S. Pinkall, A canonical metric for Möbius structures and its applications, Math. Z. 216 (1994), no. 1, 89-129. MR.1273468 (95b:53017)

[12] L. Keen, C. Series, Pleating invariants for punctured torus groups, Topology 43 (2004), no. 2, 447-491. MR2052972 (2005f:30077)

[13] L. Keen, C. Series, How to bend pairs of punctured tori, Lipa's legacy (New York, 1995), 359-387, Contemp. Math., 211, Amer. Math. Soc., Providence, RI, 1997. MR1476997 (98m:30063) 
[14] I. Rivin, On geometry of convex polyhedra in hyperbolic 3-space, Ph.D. Thesis, Princeton, 1986.

[15] I. Rivin, A characterization of ideal polyhedra in hyperbolic 3-space, Ann. of Math. (2) 143 (1996), no. 1, 51-70. MR.1370757 (96i:52008)

[16] I. Rivin, Euclidean structures on simplicial surfaces and hyperbolic volume. Ann. of Math. (2) 139 (1994), no. 3, 553-580. MR1283870(96h:57010)

[17] C. Series, On Kerckhoff minima and pleating varieties for quasifuchsian groups, Geometriae Dedicata Vol. 88, 211-237, 2001. MR.1877217 (2002j:30066)

[18] C. Series, Limits of quasifuchsian groups with small bending, Duke J. Mathematics, to appear.

[19] H. Tanigawa, Grafting, harmonic maps and projective structures on surfaces, J. Differential Geom. 47 (1997), no. 3, 399-419. MR.1617652 (99c:32029)

[20] W.P. Thurston, The geometry and topology of 3-manifolds, Princeton University Lecture Notes, Online at http://www.msri.org/publications/books/gt3m, 1982

[21] W.P. Thurston, Earthquakes in two-dimensional hyperbolic geometry, Low-dimensional topology and Kleinian groups (Coventry/Durham, 1984), 91-112, London Math. Soc. Lecture Note Ser., 112, Cambridge Univ. Press, Cambridge, 1986.

Institute for Studies in Theoretical Physics and Mathematics (IMP), Tehran, Iran

Current address: Institute for Mathematical Sciences, Stony Brook University, Stony Brook, New York 11794-3660

E-mail address: rchamanara@math.sunysb.edu 